Supporting Information:

Probing Binding Requirements of Type I and Type II Isoforms of Inosine Monophosphate Dehydrogenase with Adenine-modified Nicotinamide Adenine Dinucleotide analogues

Liqiang Chen, Guangyao Gao, Krzysztof Felczak, Laurent Bonnac, Steven E. Patterson, Daniel Wilson, Eric M. Bennett, Hiremagalur N. Jayaram, Lizbeth Hedstrom, and Krzysztof W. Pankiewicz

Table of contents:

- HPLC profiles of tiazofurin adnine dinucleotide (TAD) analogues: S2 - S37

- HPLC profiles of mycophenolic adenine dinucleotides (MAD) analogues S38 S43 


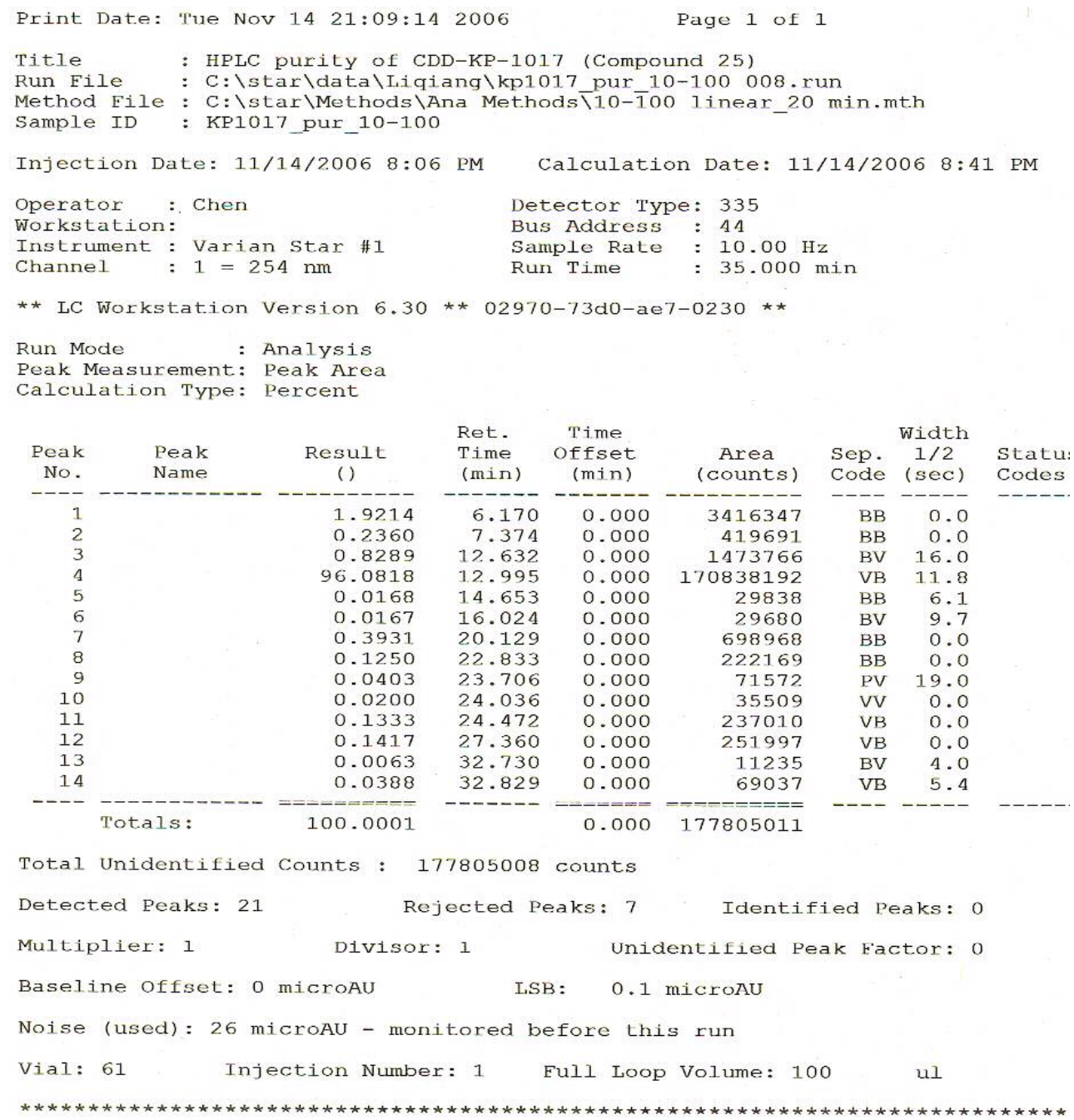


'Ilte : HPLC purlty of CDD-KP-1017 (Compound 25)

Run File : C: \star \data \Liqiang $\backslash k p 1017$ pur 10-100 008.run

Method File : C: \star\Methods \Ana Methods $\backslash 10-\overline{1} 00$ linear 20 min.mth

Sample ID : KP1017_pur_10-100

Injection Date: 11/14/2006 8:06 PM Calculation Date: 11/14/2006 8:41 PM

Operator : Chen

Workstation:

Instrument : Varian Star \#1

Detector Type: 335

Channel : $1=254 \mathrm{~nm}$

Bus Address : 44

Sample Rate : $10.00 \mathrm{~Hz}$

Run Time: $35.000 \mathrm{~min}$

** LC Workstation Version 6.30 ** 02970-73d0-ae7-0230 **

Chart Speed $=0.56 \mathrm{~cm} / \mathrm{min} \quad$ Attenuation $=40 \quad$ Zero Offset $=3 \%$

Start Time $=0.000$ min End Time $=35.000 \mathrm{~min}$ Min $/ \mathrm{Tick}=1.00$

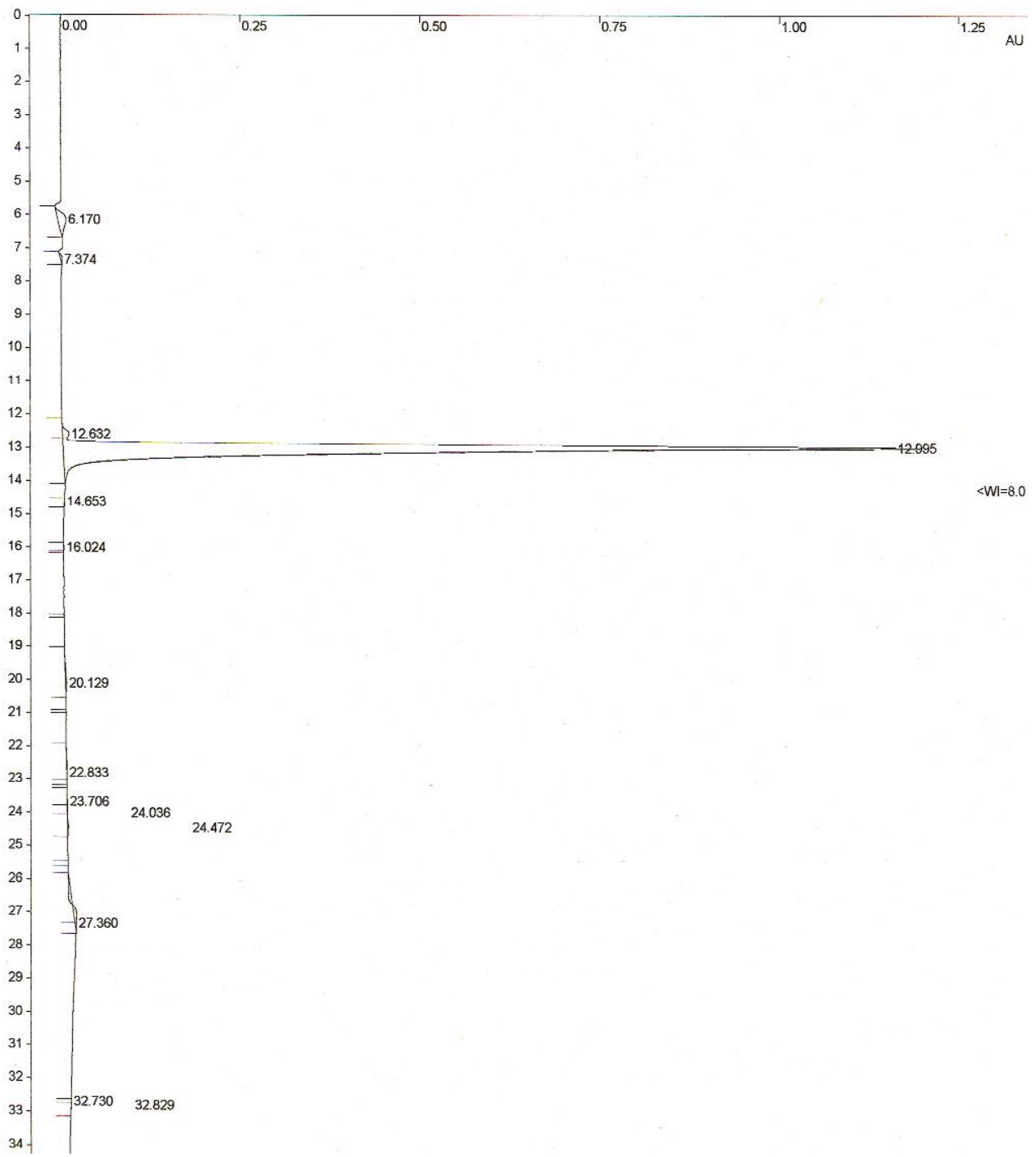




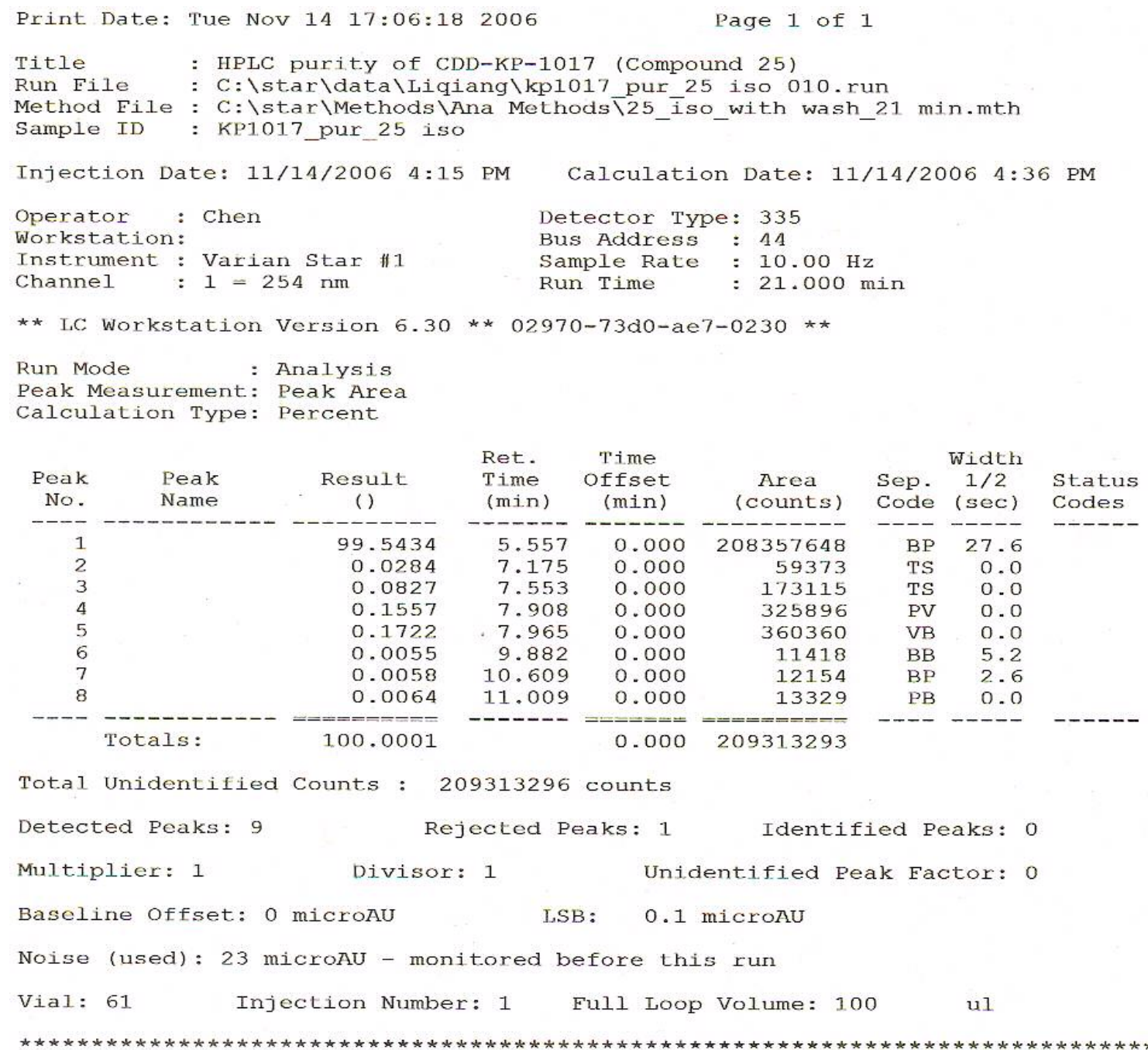


'l'ıtle : HPLC purity of CDD-KP-1017 (Compound 25)

Run File : C: \star\data\Liqiang \kp1017 pur 25 iso 010.run

Method File : C: \star\Methods\Ana Methods $\backslash 25$ iso_with wash 21 min.mth

Sample ID : KP1017_pur_25 iso.

Injection Date: 11/14/2006 4:15 PM Calculation Date: 11/14/2006 4:36 PM

Operator : Chen

Workstation:

Instrument : Varian star \#1

Detector Type: 335

Bus Address : 44

Sample Rate : $10.00 \mathrm{~Hz}$

Channel : $1=254 \mathrm{~nm}$

Run Time : $21.000 \mathrm{~min}$

** LC Workstation Version 6.30 ** 02970-73d0-ae7-0230 **

Chart Speed $=0.93 \mathrm{~cm} / \mathrm{min} \quad$ Attenuation $=23 \quad$ Zero offset $=3 \%$

Start Time $=0.000 \mathrm{~min}$ End Time $=21.000 \mathrm{~min}$ Min $/ \mathrm{Tick}=1.00$

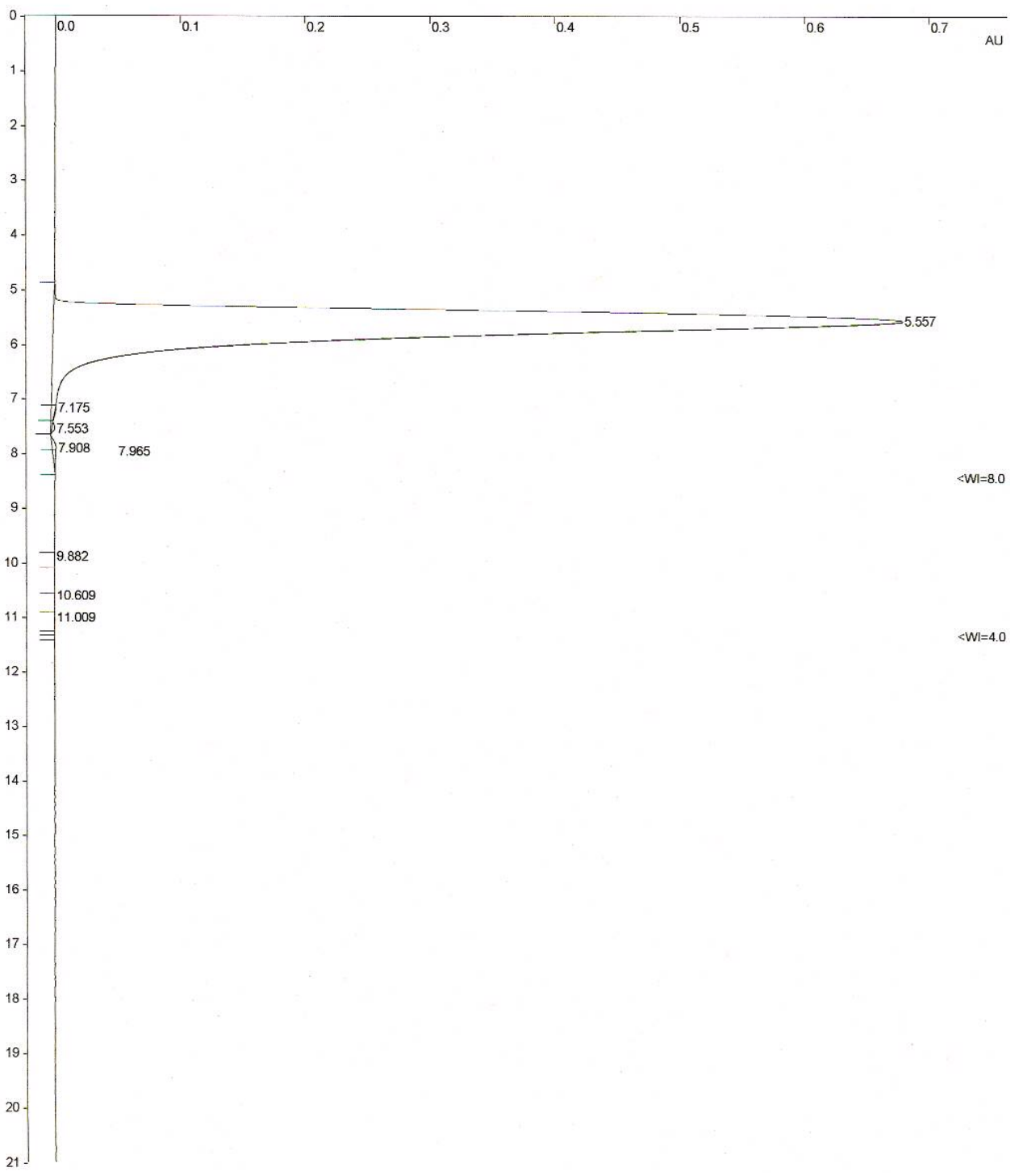




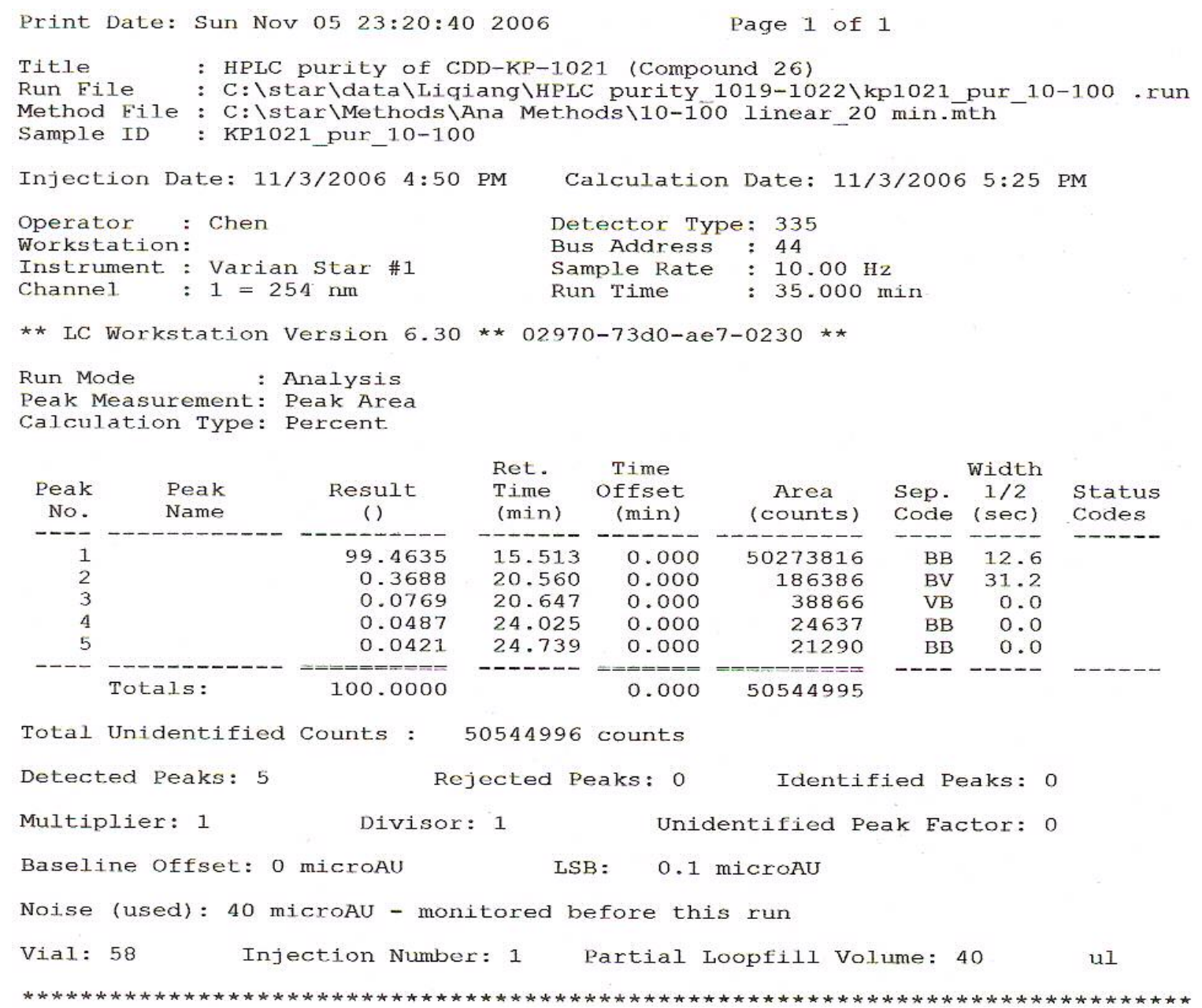


Title : HPLC purity of CDD-KP-1021 (Compound 26)

Run File : C: \star\data\Liqiang\HPLC purity 1019-1022\kp1021 pur 10-100 .run

Method File : C: \star \Methods \Ana Methods $\backslash 10-1 \overline{0} 0$ linear 20 min.mth

Sample ID : KP1021_pur_10-100

Injection Date: 11/3/2006 4:50 PM Calculation Date: 11/3/2006 5:25 PM

$\begin{array}{ll}\text { Operator : Chen } & \text { Detector Type: } 335 \\ \text { Workstation: } & \text { Bus Address : } 44 \\ \text { Instrument : Varian Star \#1 } & \text { Sample Rate : } 10.00 \mathrm{~Hz} \\ \text { Channel : } 1=254 \mathrm{~nm} & \text { Run Time }: 35.000 \mathrm{~min}\end{array}$

** LC Workstation Version $6.30 \star \star ~ 02970-73 d 0-a e 7-0230 * \star$

Chart Speed $=0.56 \mathrm{~cm} / \mathrm{min} \quad$ Attenuation $=11 \quad$ Zero offset $=5 \%$

Start Time $=0.000 \mathrm{~min}$ End Time $=35.000 \mathrm{~min} \mathrm{Min} / \mathrm{Tick}=1.00$

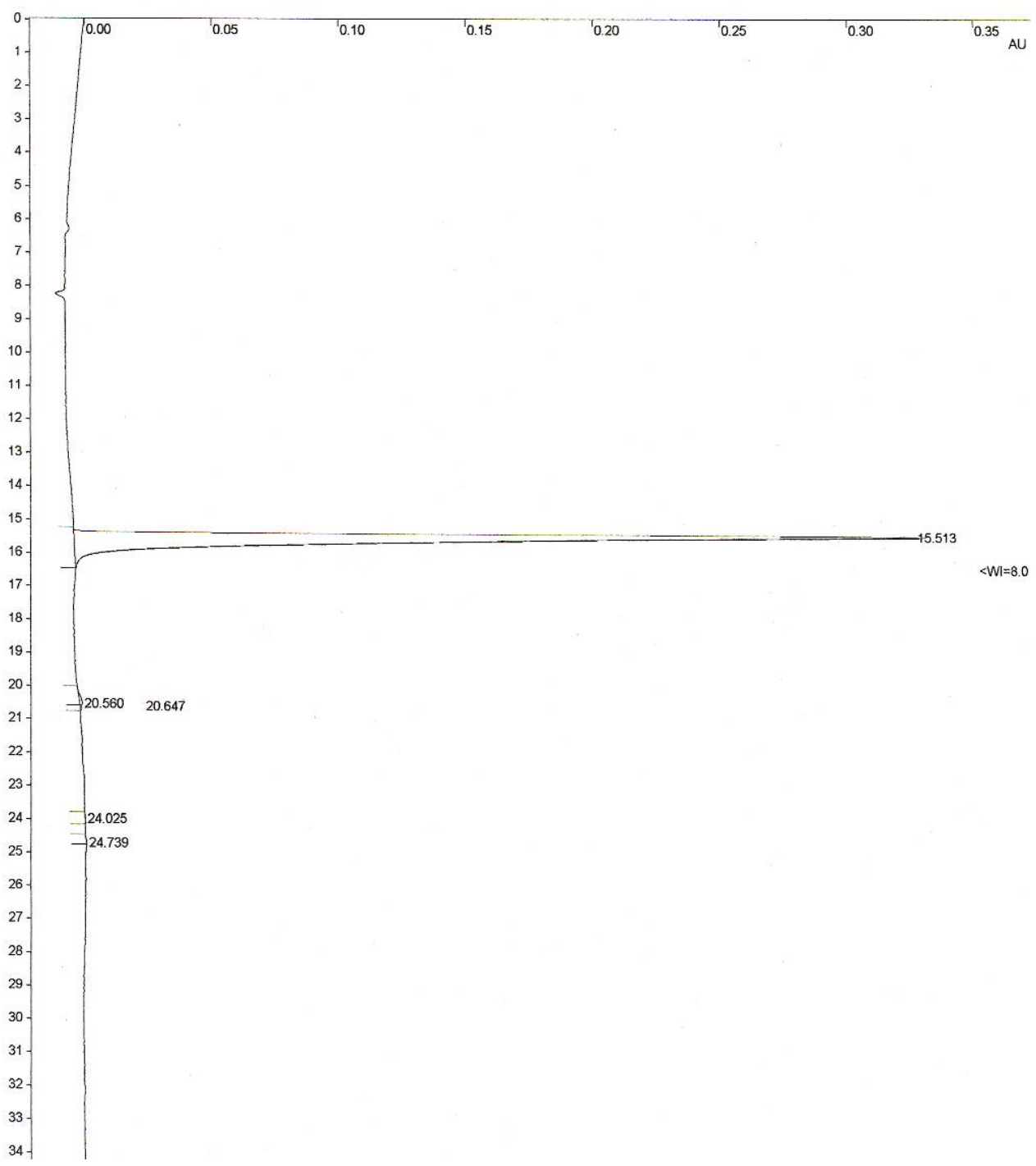




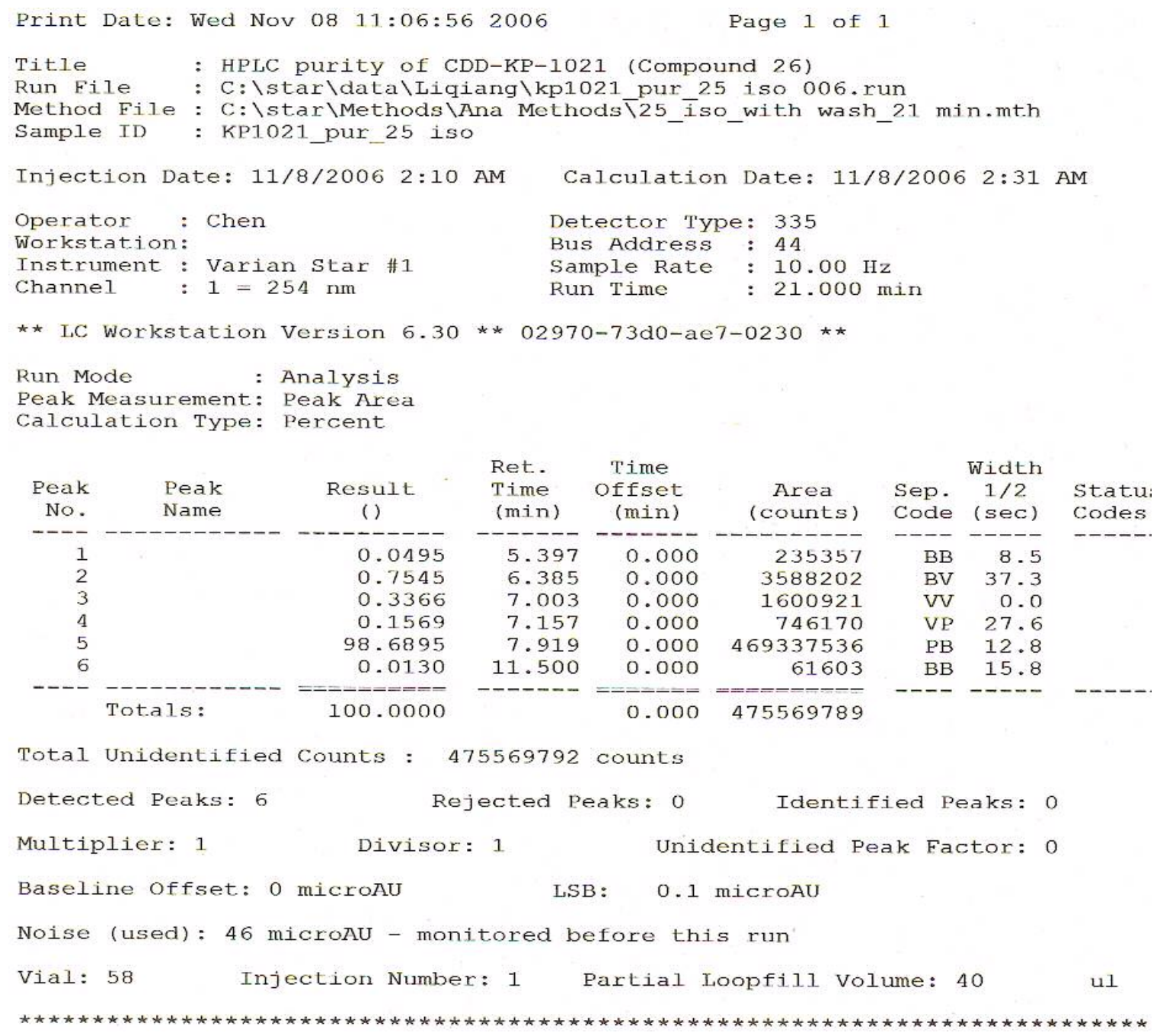


Title : HPLC purity of CDD-KP-1021 (Compound 26)

Run File : C: \star\data\Liqiang $\backslash k p 1021$ pur 25 iso 006.run

Method File : C: \star $\backslash$ Methods $\backslash$ Ana Methods $\backslash 25$ iso with wash 21 min.mth

Sample ID : KP1021_pur_25 iso

Injection Date: 11/8/2006 2:10 AM Calculation Date: 11/8/2006 2:31 AM

Operator : Chen

Workstation:

Instrument : Varian Star \#1

Detector Type: 335

Channel : $1=254 \mathrm{~nm}$

Bus Address : 44

Sample Rate : $10.00 \mathrm{~Hz}$

Run Time : $21.000 \mathrm{~min}$

** LC Workstation Version 6.30** 02970-73d0-ae7-0230 **

Chart Speed $=0.93 \mathrm{~cm} / \mathrm{min} \quad$ Attenuation $=99 \quad$ Zero offset $=2 \%$

Start Time $=0.000 \mathrm{~min}$ End Time $=21.000 \mathrm{~min}$ Min $/ \mathrm{Tick}=1.00$

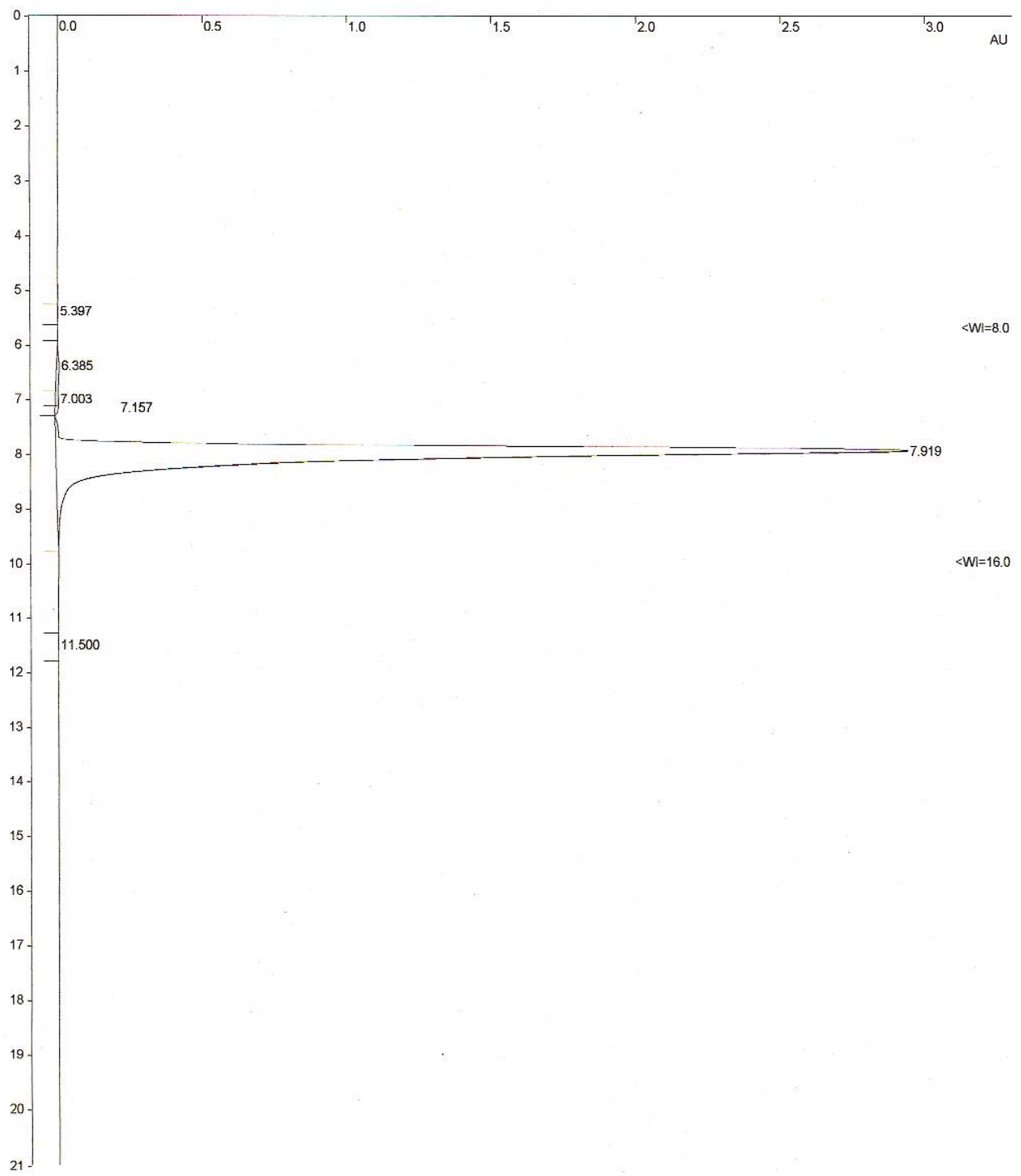




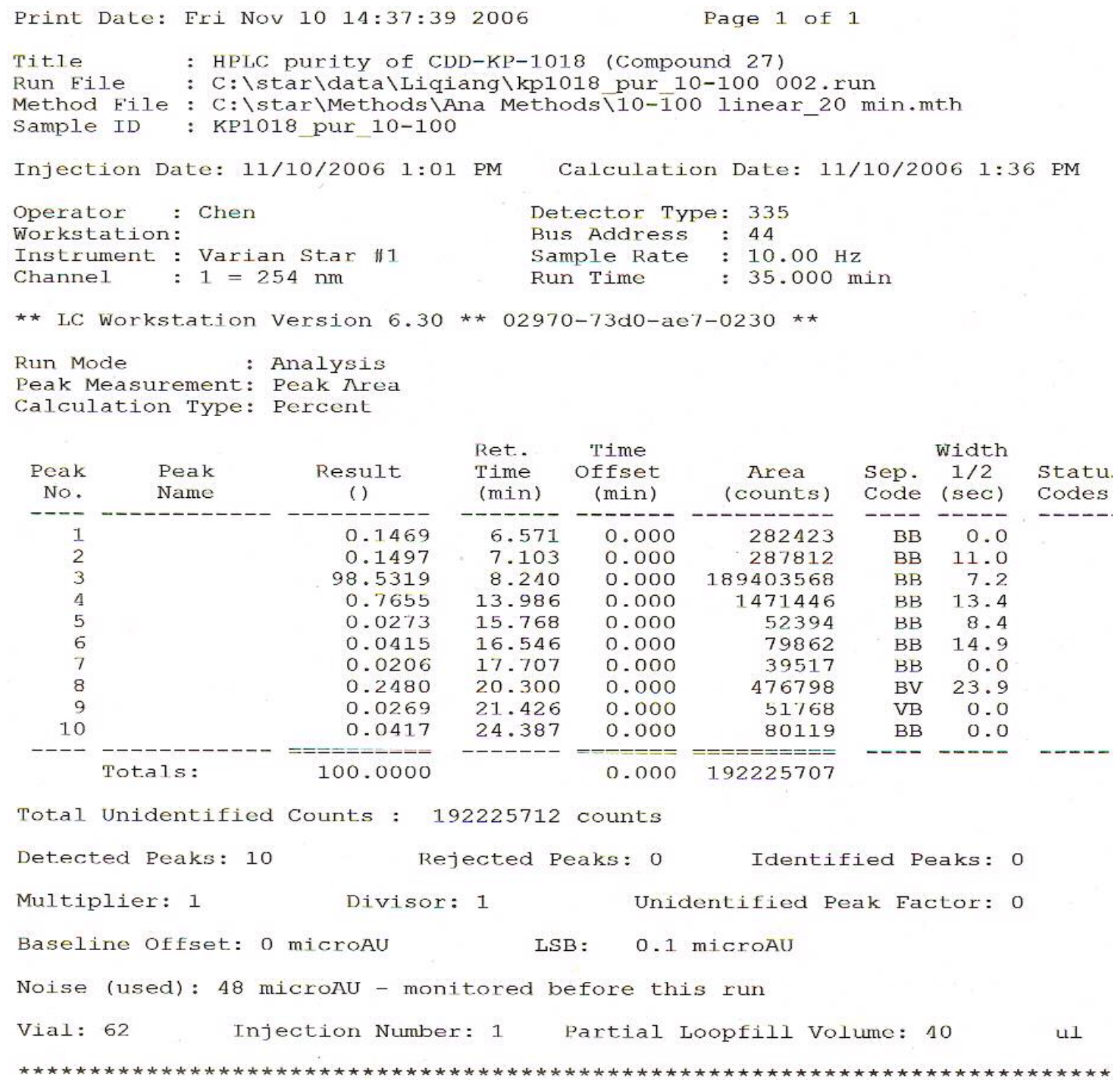


Title : HPLC purity of CDD-KP-1018 (Compound 27)

Run File : C: \star \data \Liqiang \kp1018 pur 10-100 002.run

Method File : C: \star\Methods \Ana Methods $\backslash 10-\overline{1} 00$ linear 20 min.mth

Sample ID : KP1018_pur_10-100

Injection Date: 11/10/2006 1:01 PM Calculation Date: 11/10/2006 1:36 PM

Operator : Chen

Workstation:

Instrument : Varian Star \#1

Detector Type: 335

Channel

: $1=254 \mathrm{~nm}$

Bus Address : 44

Sample Rate : $10.00 \mathrm{~Hz}$

Run Time : $35.000 \mathrm{~min}$

** LC Workstation Version 6.30 ** 02970-73d0-ae7-0230 **

Chart Speed $=0.56 \mathrm{~cm} / \mathrm{min} \quad$ Attenuation $=57 \quad$ Zero offset $=2 \%$

Start Time $=0.000 \mathrm{~min}$ End Time $=35.000 \mathrm{~min} \mathrm{Min} / \mathrm{Tick}=1.00$

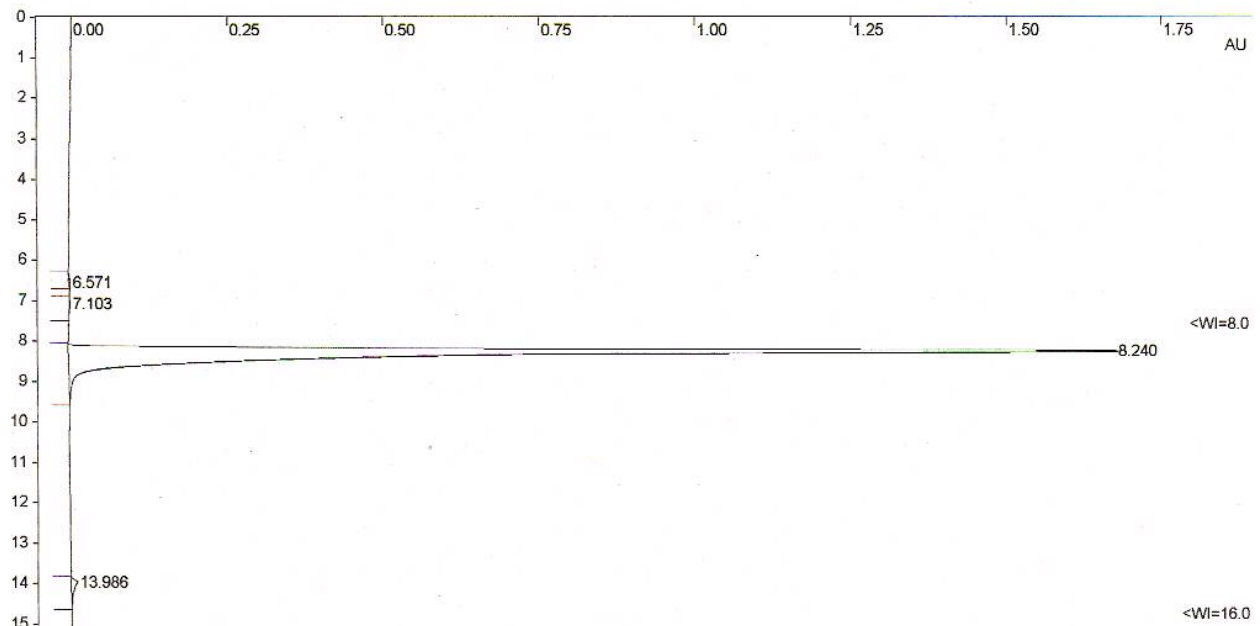




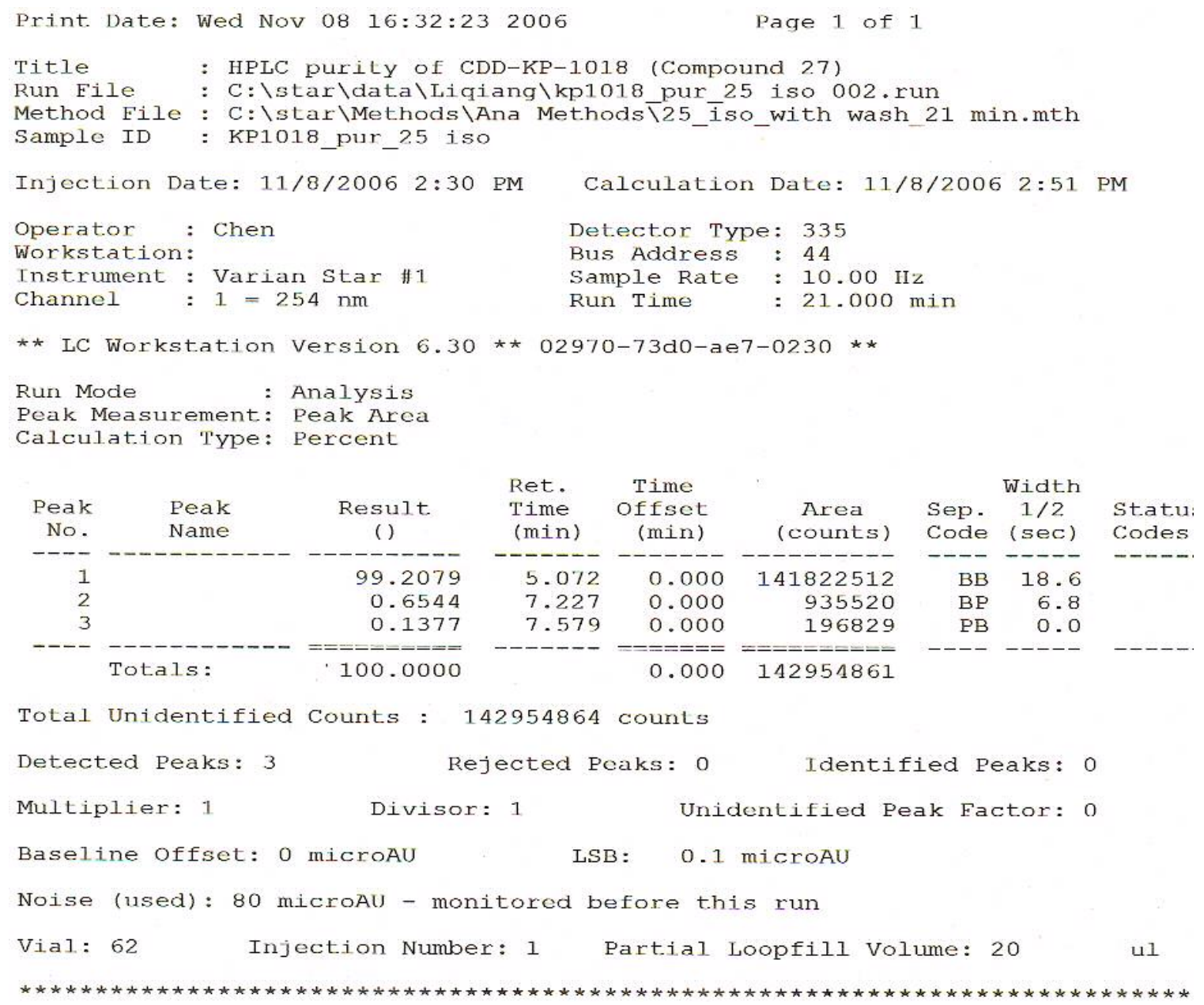


Title : HPLC purity of CDD-KP-1018 (Compound 27)

Run File : C: \star \data \Liqiang $\backslash k p 1018$ pur 25 iso 002. run

Method File : C: \star\Methods \Ana Methods $\backslash 25$ iso with wash 21 min.mth

Sample ID : KP1018_pur_25 iso

Injection Date: 11/8/2006 2:30 PM Calculation Date: 11/8/2006 2:51 PM

$\begin{array}{ll}\text { Operator : Chen } & \text { Detector Type: } 335 \\ \text { Workstation: } & \text { Bus Address : } 44 \\ \text { Instrument : Varian Star \#1 } & \text { Sample Rate : } 10.00 \mathrm{~Hz} \\ \text { Channel }: 1=254 \mathrm{~nm} & \text { Run Time }: 21.000 \mathrm{~min}\end{array}$

** LC Workstation Version 6.30 ** 02970-73d0-ae7-0230 **

Chart Speed $=0.93 \mathrm{~cm} / \mathrm{min} \quad$ Attenuation $=24 \quad$ Zero offset $=3 \%$

Start Time $=0.000 \mathrm{~min}$ End Time $=21.000 \mathrm{~min}$ Min $/ \mathrm{Tick}=1.00$

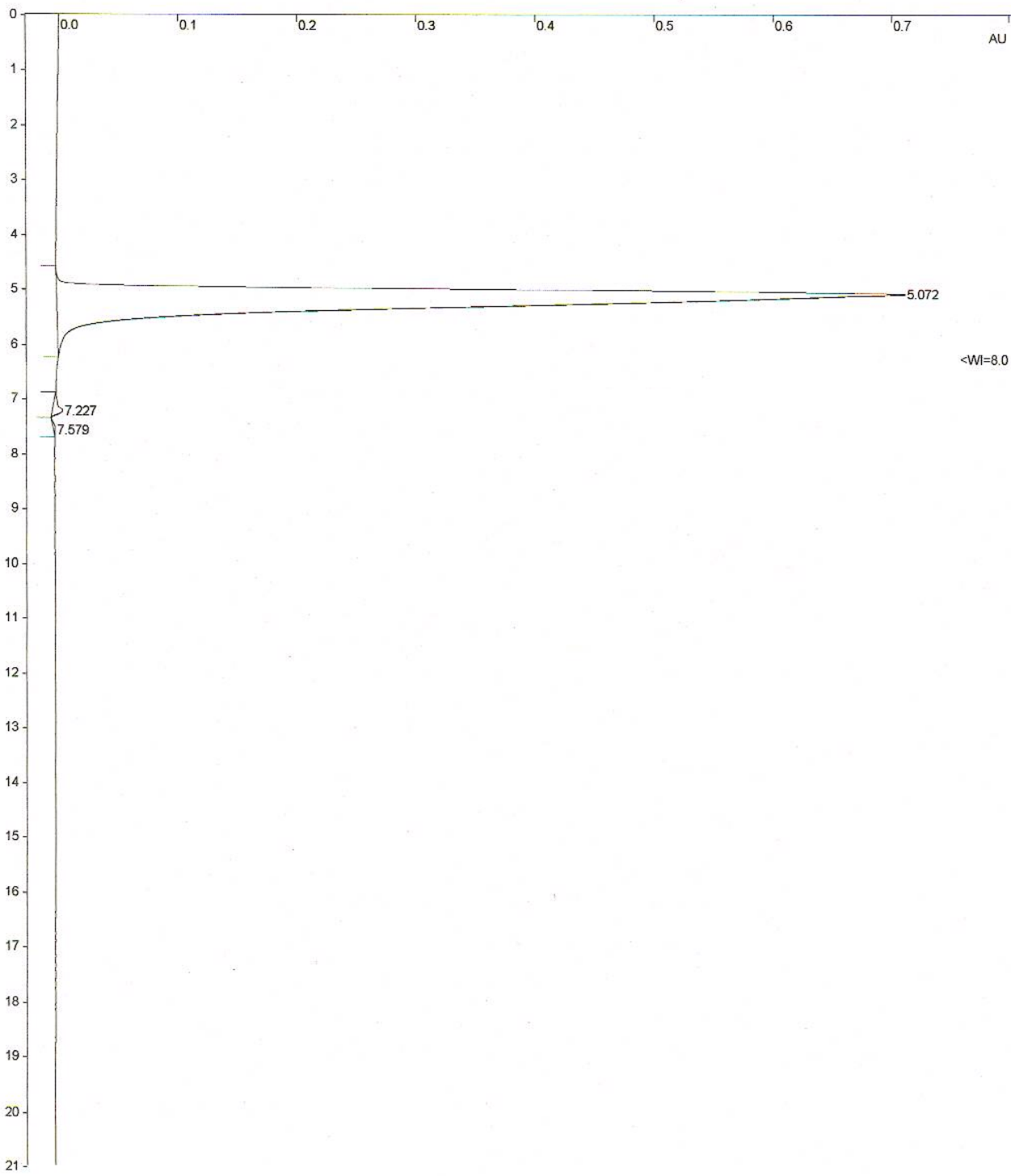




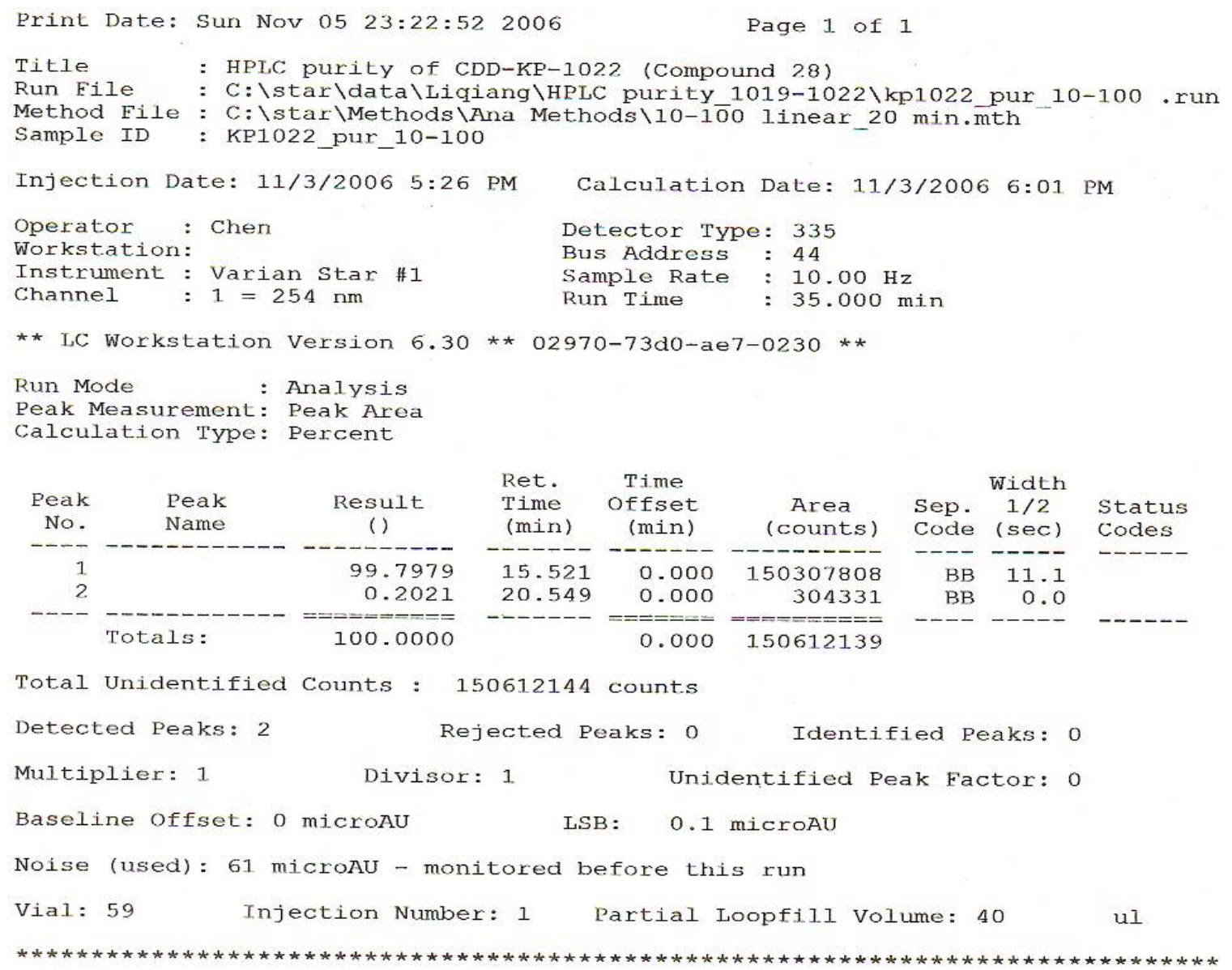


'l'Itle : HPLC purity of CDD-KP-1022 (Compound 28)

Run File : C: \star\data \Liqiang \HPLC purity 1019-1022\kp1022 pur 10-100 .run

Method File : C: \star\Methods \Ana Methods $\backslash 10-100$ linear 20 min.mth

Sample ID : KP1022_pur_10-100

Injection Date: 11/3/2006 5:26 PM Calculation Date: 11/3/2006 6:01 PM

$\begin{array}{ll}\text { Operator : Chen } & \text { Detector Type: } 335 \\ \text { Workstation: } & \text { Bus Address : } 44 \\ \text { Instrument : Varian Star \#1 } & \text { Sample Rate }: 10.00 \mathrm{~Hz} \\ \text { Channel }: 1=254 \mathrm{~nm} & \text { Run Time }: 35.000 \mathrm{~min}\end{array}$

** LC Workstation Version $6.30 *$ 02970-73d0-ae7-0230 **

Chart Speed $=0.56 \mathrm{~cm} / \mathrm{min} \quad$ Attenuation $=36 \quad$ Zero offset $=3 \%$

Start $\mathrm{Time}=0.000 \mathrm{~min}$ End Time $=35.000 \mathrm{~min}$ Min $/ \mathrm{Tick}=1.00$

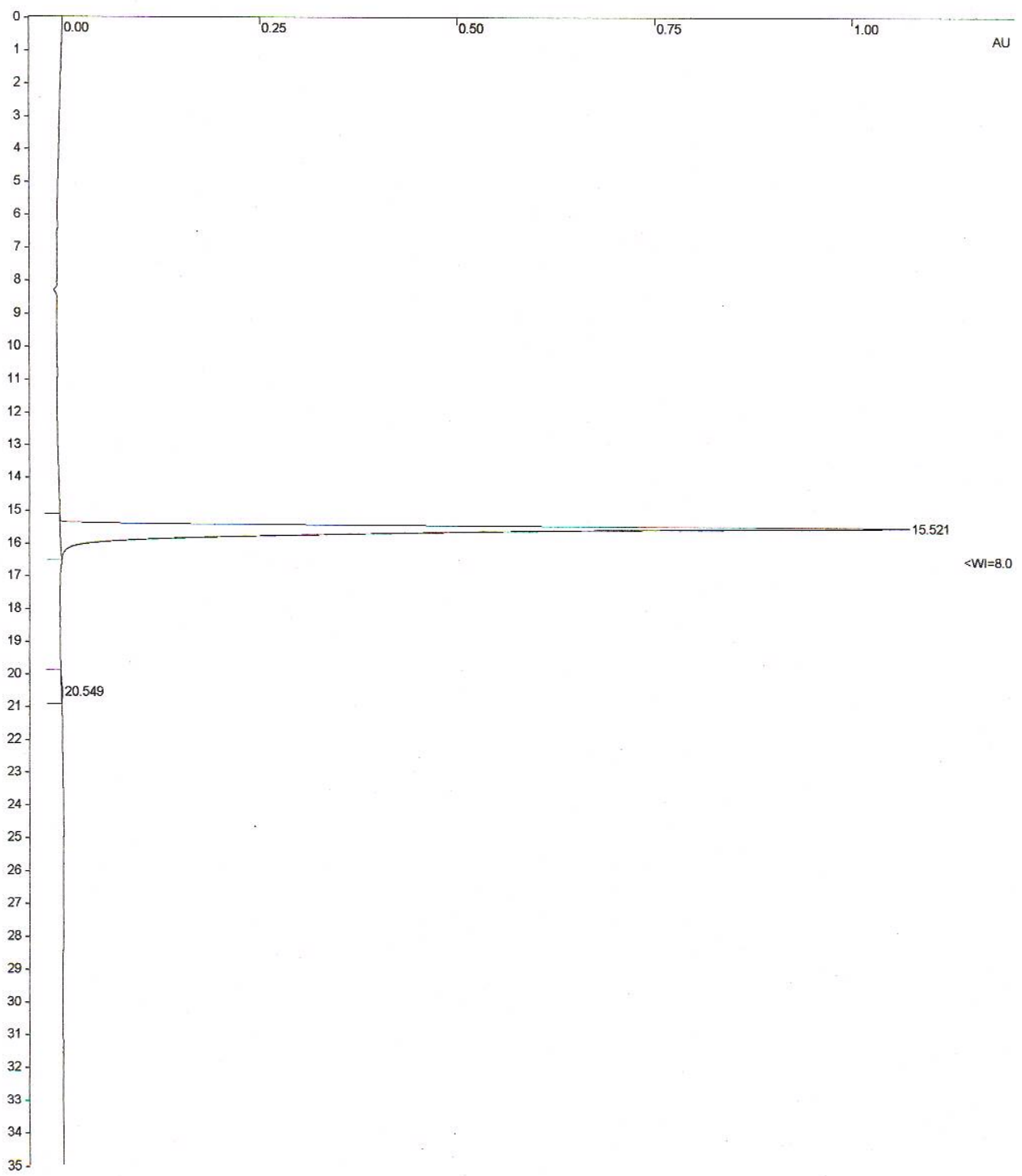




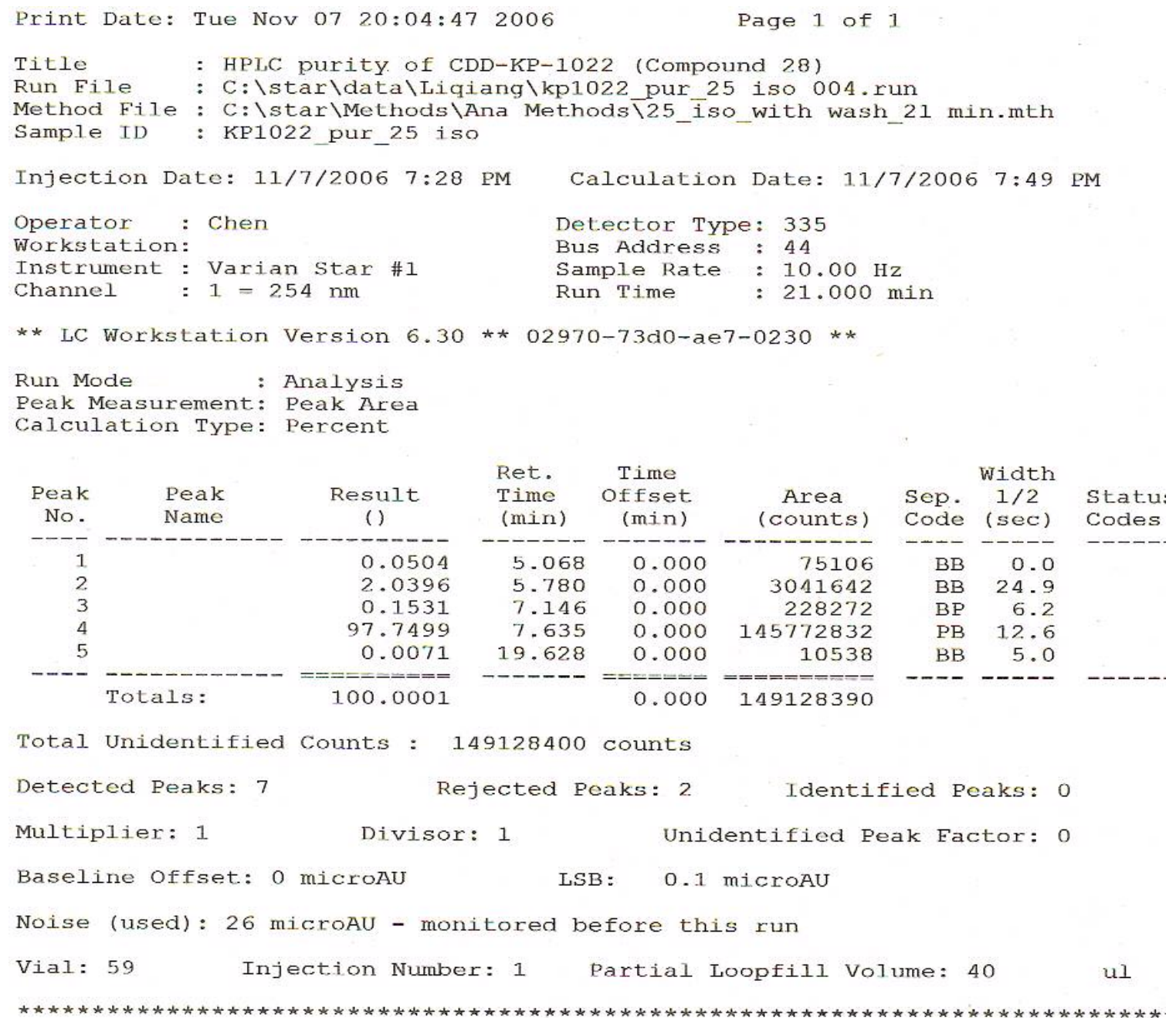


'l'1t1e : HPLC purıty of CDD-KP-1022 (Compound 28)

Run File : C: \star \data\Liqiang $\backslash k p 1022$ pur 25 iso 004.run

Method File : C: \star \Methods $\backslash$ Ana Methods $\backslash 25$ iso with wash 21 min.mth

Sample ID : KP1022_pur_25 iso

Injection Date: 11/7/2006 7:28 PM Calculation Date: 11/7/2006 7:49 PM

Operator : Chen

Workstation:

Instrument : Varian star \#1

Channel : $1=254 \mathrm{~nm}$

** LC Workstation Version 6.30** 02970-73d0-ae7-0230 **

Chart Speed $=0.93 \mathrm{~cm} / \mathrm{min} \quad$ Attenuation $=30 \quad$ Zero Offset $=2 \%$

Start Time $=0.000 \mathrm{~min}$ End Time $=21.000 \mathrm{~min}$ Min $/ \mathrm{Tick}=1.00$

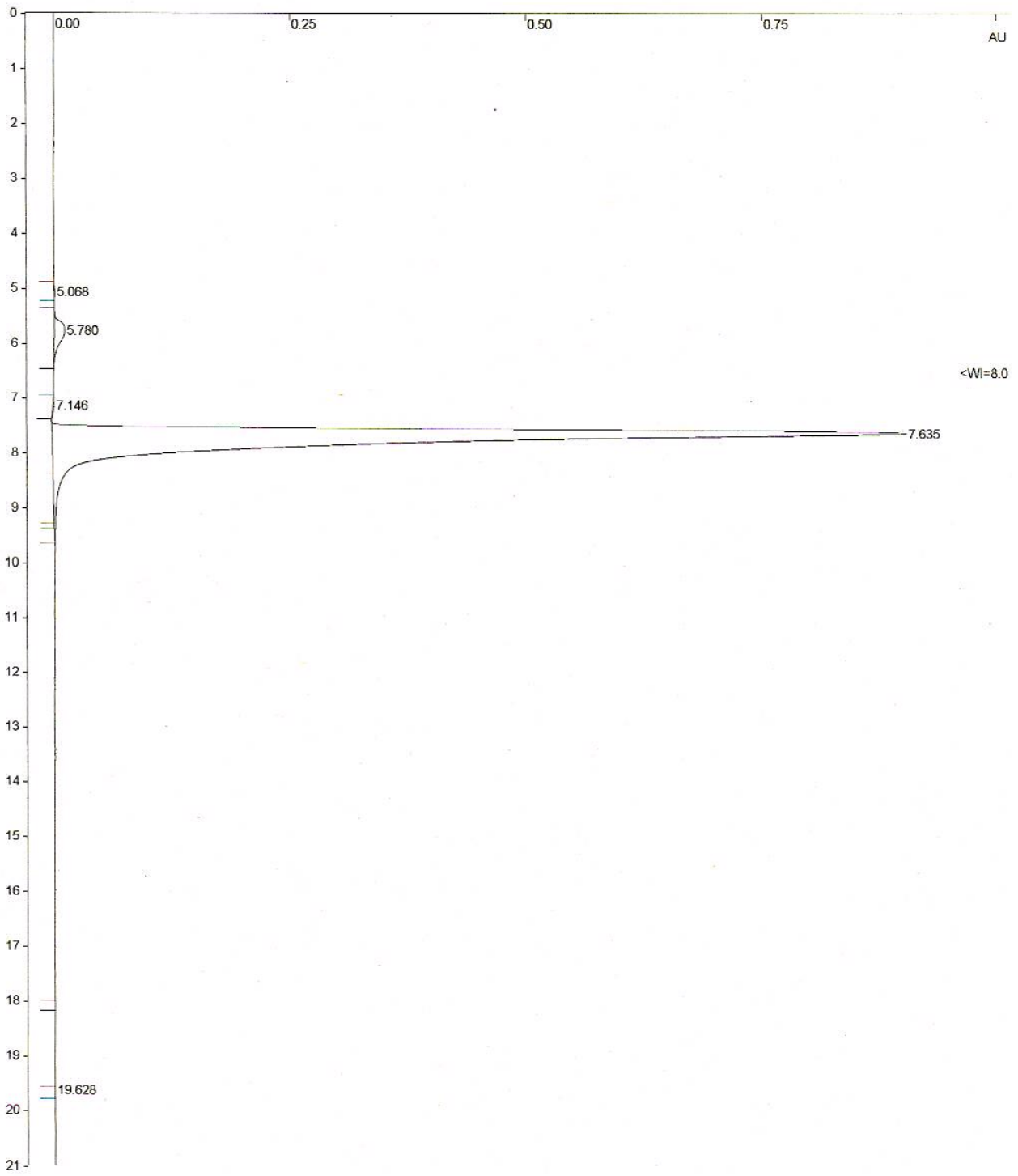




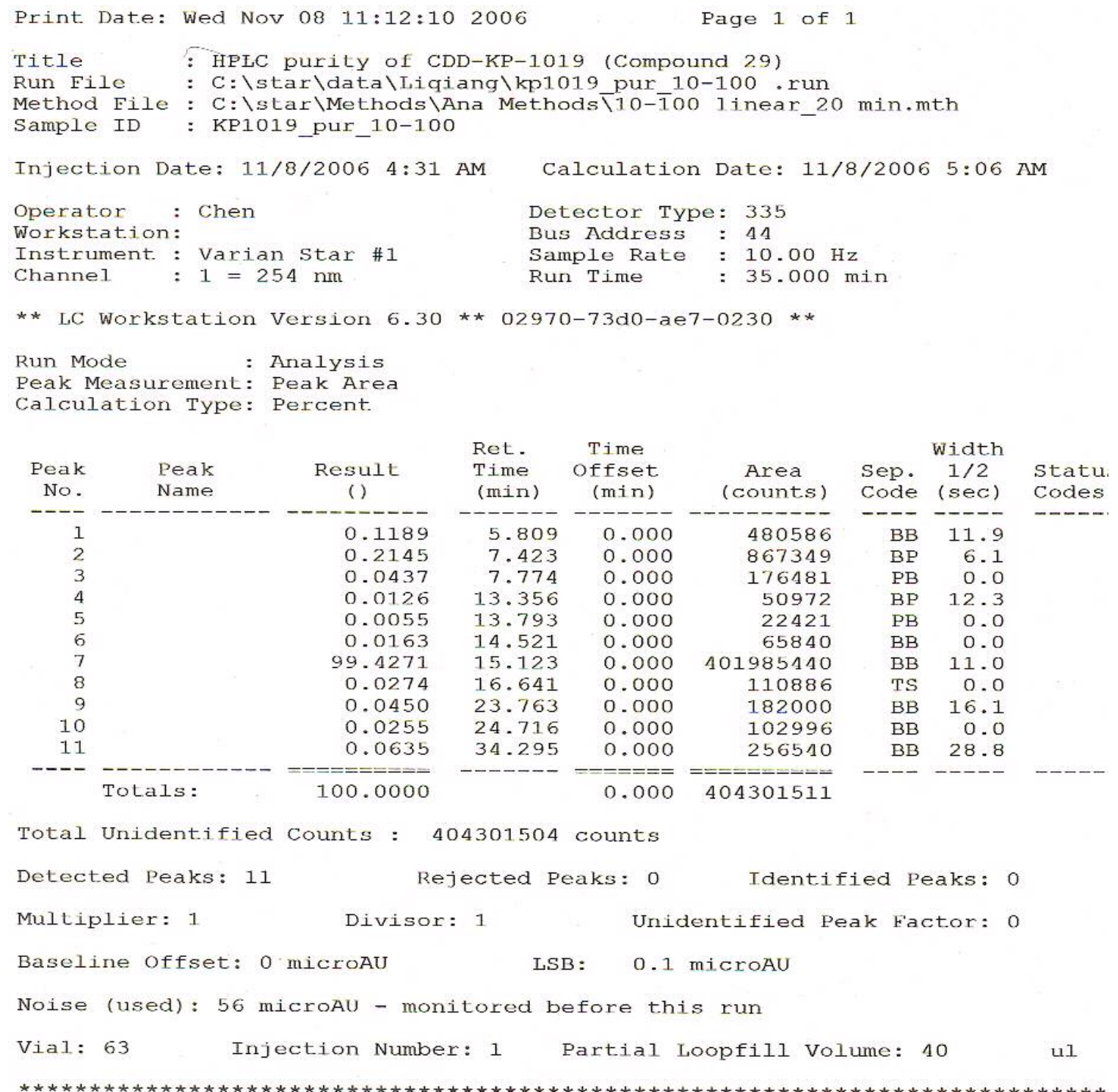


Title : HPLC purity of CDD-KP-1019 (Compound 29)

Run File : C: \star \data \Liqiang $\backslash k p 1019$ pur 10-100 .run

Method File : C: \star\Methods\Ana Methods $\backslash 10-\overline{1} 00$ linear 20 min.mth

Sample ID : KP1019_pur_10-100

Injection Date: 11/8/2006 4:31 AM Calculation Date: 11/8/2006 5:06 AM

Operator : Chen

Workstation:

Instrument : Varian star \#1

Detector Type: 335

Channel

: $1=254 \mathrm{~nm}$

Bus Address : 44

Sample Rate : $10.00 \mathrm{~Hz}$

** LC Workstation Version 6.30 ** 02970-73d0-ae7-0230 * *

Chart Speed $=0.56 \mathrm{~cm} / \mathrm{min} \quad$ Attenuation $=96 \quad$ Zero Offset $=2 \%$

Start Time $=0.000 \mathrm{~min}$ End Time $=35.000 \mathrm{~min}$ Min $/ \mathrm{Tick}=1.00$

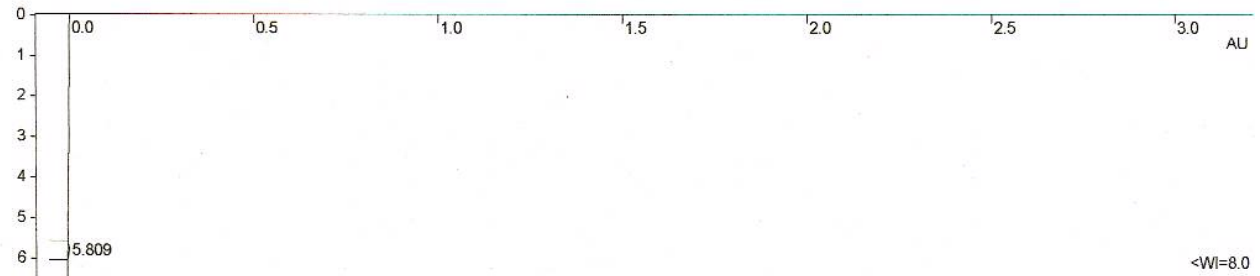

$7.423 \quad 7.774$

$10-$

11

12.

13. -

$-13.356 \quad 13.793$

$14-$

-14.521

16 -

17. -16.641

18.

19.

$20-$

21.

$22-$

$24-23.763$

$25-24.716$

$<W \mid=16.0$ 


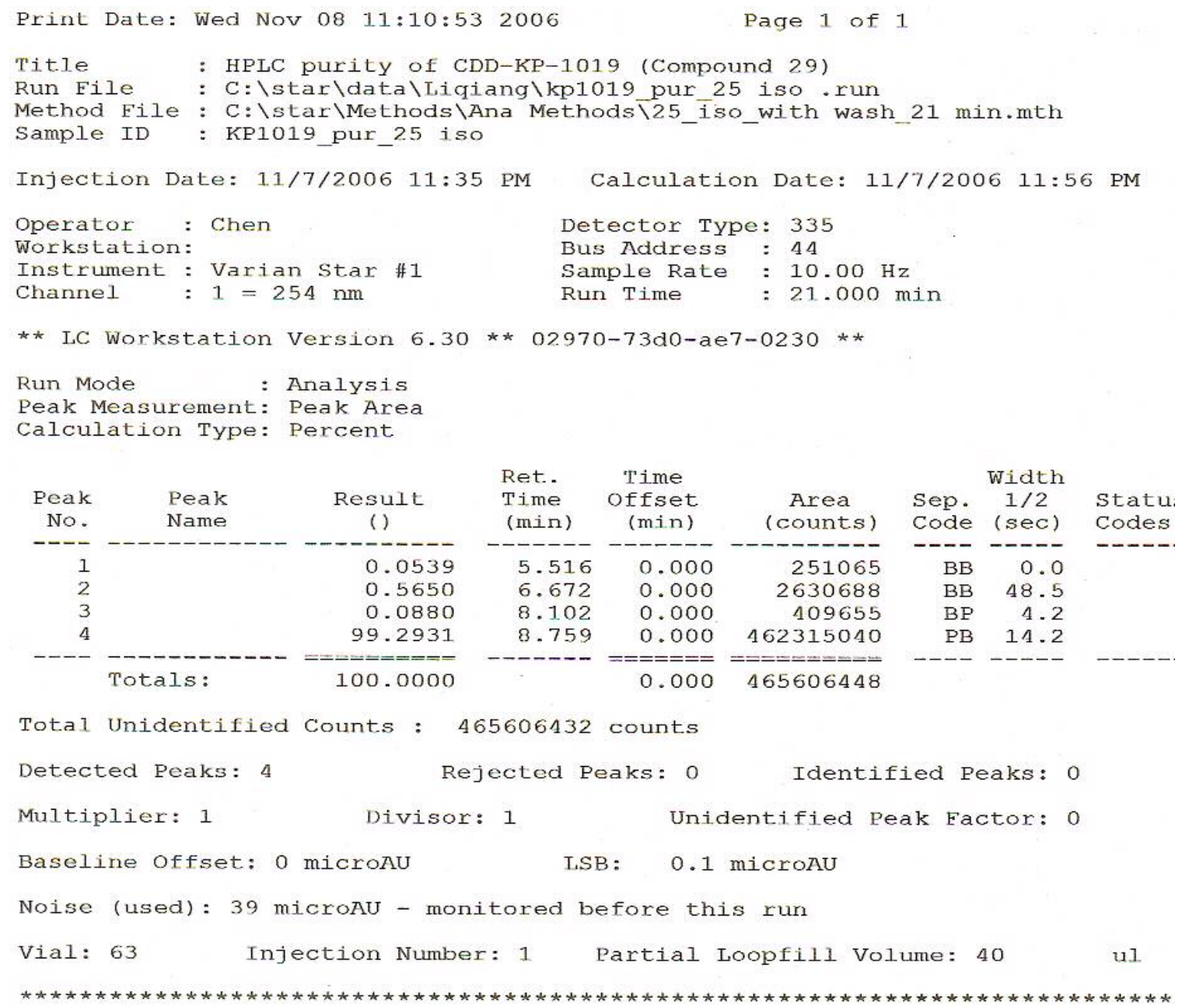


'I'lte : HPLC purıty of CDD-KP-1019 (Compound 29)

Run File : C: \star\data\Liqiang $\backslash k p 1019$ pur 25 iso .run

Method File : C: \star \Methods \Ana Methods $\backslash 25$ iso with wash 21 min.mth

Sample ID : KP1019_pur_25 iso

Injection Date: 11/7/2006 11:35 PM Calculation Date: 11/7/2006 11:56 PM

Operator : Chen

Workstation:

Instrument : Varian Star \#1

Detector Type: 335

Bus Address : 44

Channel : $1=254 \mathrm{~nm}$

Sample Rate : $10.00 \mathrm{~Hz}$

Run Time : $21.000 \mathrm{~min}$

** LC Workstation Version 6.30 ** 02970-73d0-ae7-0230 **

Chart Speed $=0.93 \mathrm{~cm} / \mathrm{min} \quad$ Attenuation $=86 \quad$ Zero offset $=2 \%$

Start Time $=0.000 \mathrm{~min}$ End Time $=21.000 \mathrm{~min}$ Min $/ \mathrm{Tick}=1.00$

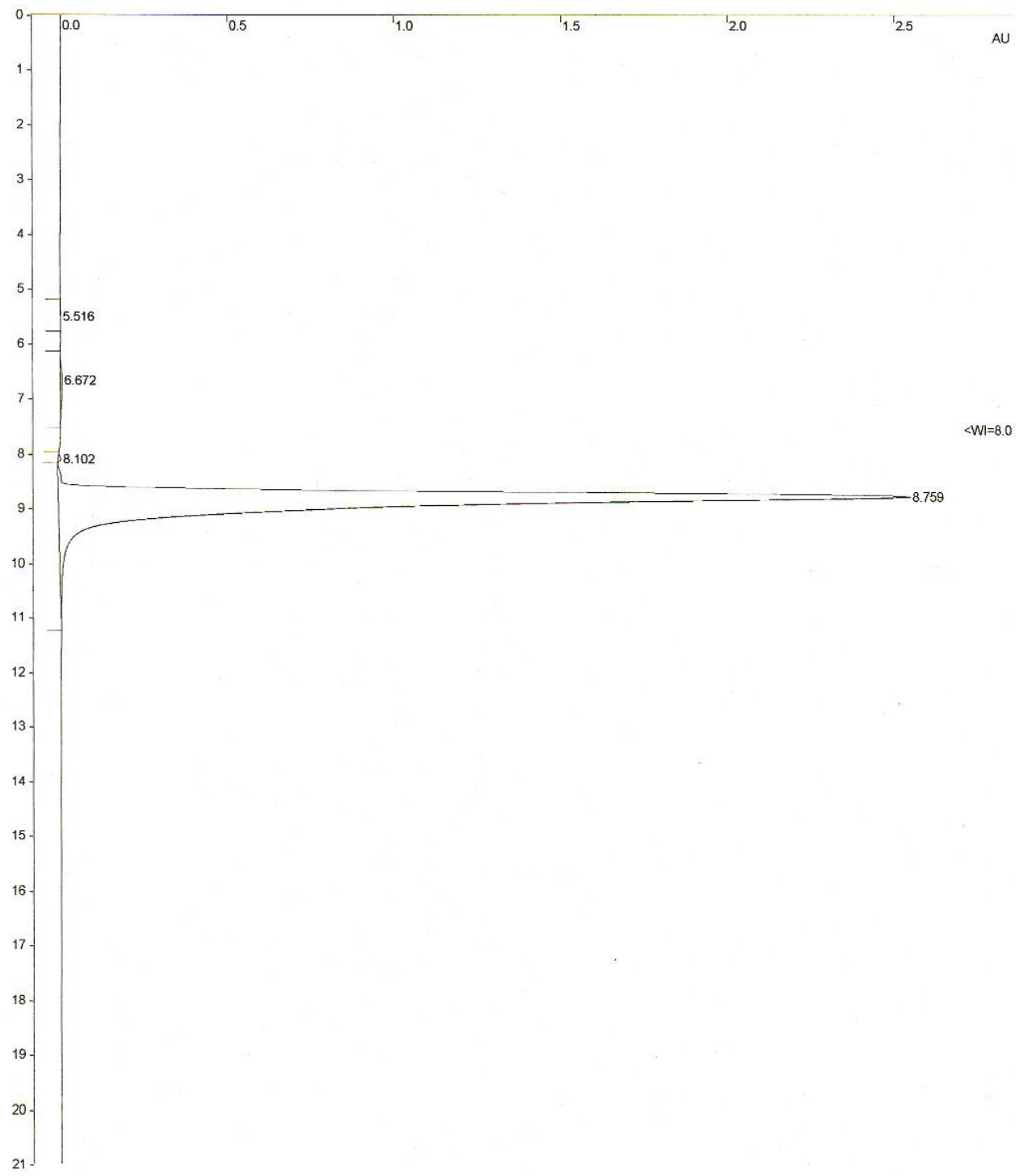




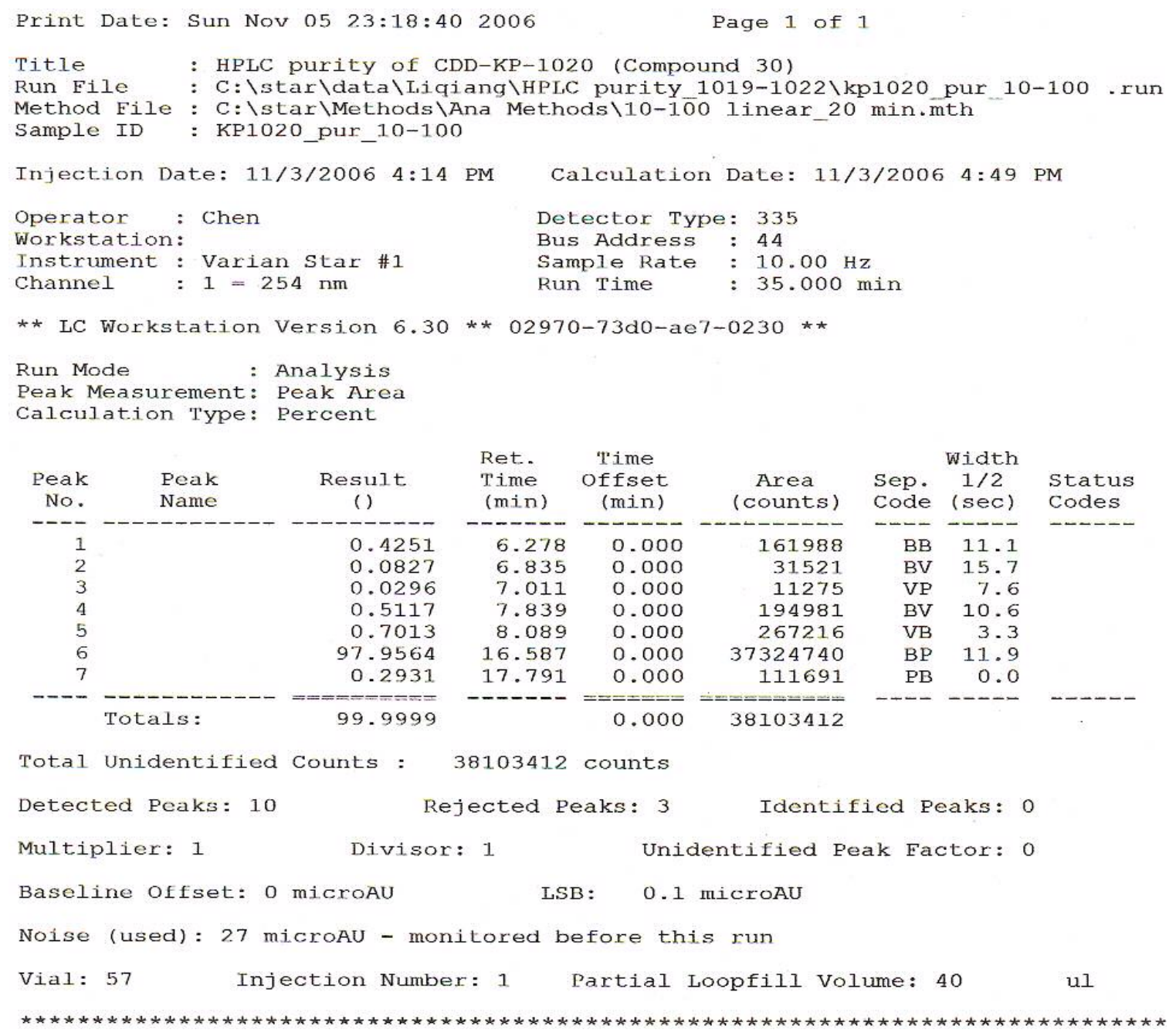


Title : HPLC purity of CDD-KP-1020 (Compound 30)

Run File : C: \star\data\Liqiang\HPLC purity_1019-1022\kp1020_pur_10-100 .run

Method File : C: \star \Methods \Ana Methods $\backslash 10-1 \overline{0} 0$ linear_20 min. $\bar{m}$ th

Sample ID : KP1020_pur_10-100

Injection Date: 11/3/2006 4:14 PM Calculation Date: 11/3/2006 4:49 PM

$\begin{array}{ll}\text { Operator : Chen } & \text { Detector Type: } 335 \\ \text { Workstation: } & \text { Bus Address : } 44 \\ \text { Instrument : Varian Star \#1 } & \text { Sample Rate }: 10.00 \mathrm{~Hz} \\ \text { Channel }: 1=254 \mathrm{~nm} & \text { Run Time }: 35.000 \mathrm{~min}\end{array}$

** LC Workstation Version 6.30 ** 02970-73d0-ae7-0230 **

Chart Speed $=0.56 \mathrm{~cm} / \mathrm{min} \quad$ Attenuation $=8 \quad$ Zero offset $=7 \%$

Start Time $=0.000 \mathrm{~min}$ End Time $=35.000 \mathrm{~min} \mathrm{Min} / \mathrm{Tick}=1.00$

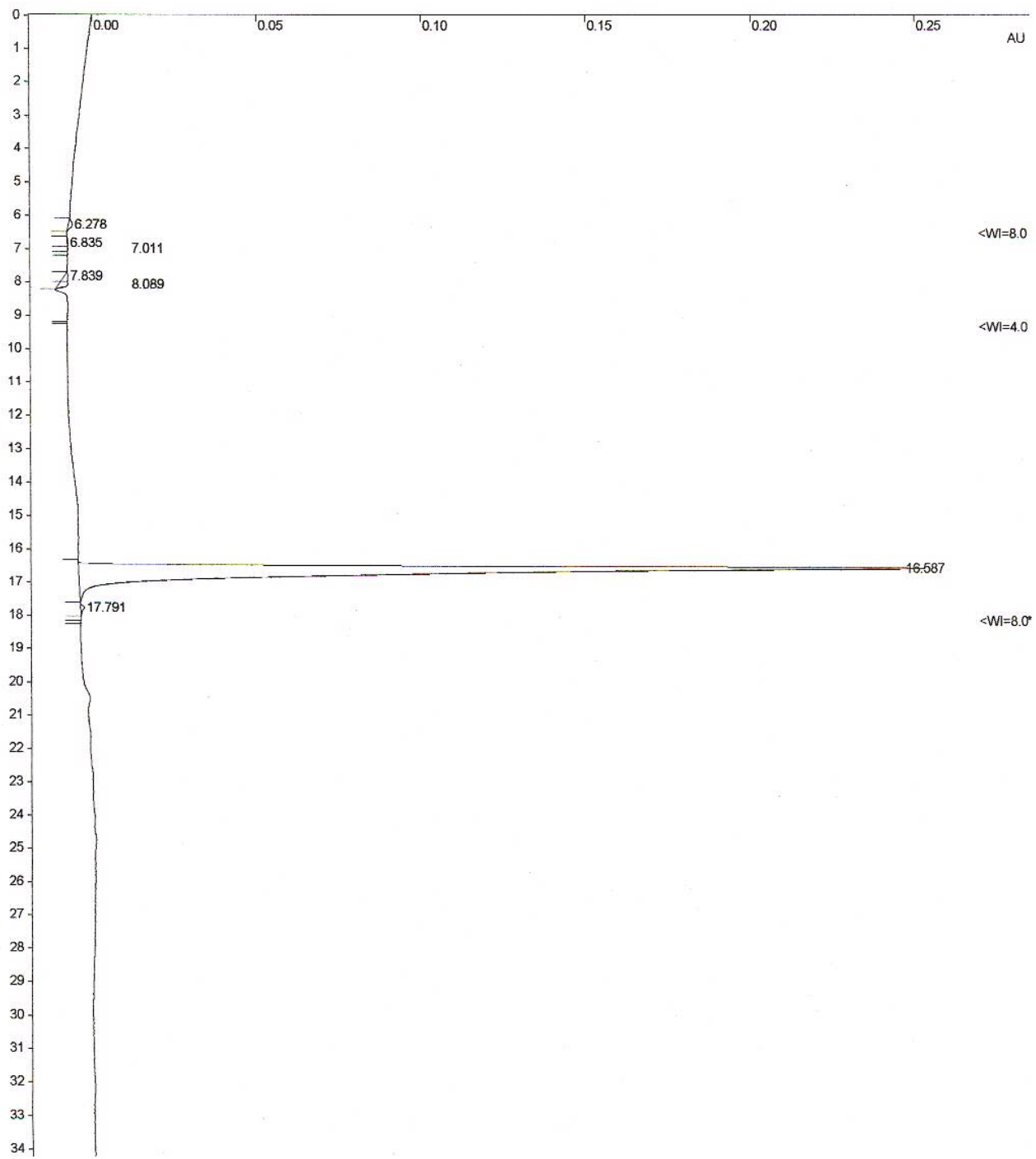




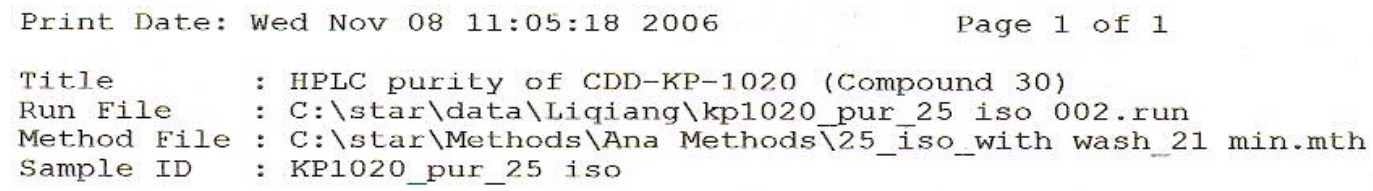


I'itıe : HPLC purity of CDD-KP-1020 (Compound 30)

Run File : C: \star \data \Liqiang $\backslash k p 1020$ pur 25 iso 002.run

Method File : C: \star $\backslash$ Methods $\backslash$ Ana Methods $\backslash 25$ iso with wash 21 min.mth

Sample ID : KP1020_pur_25 iso

Injection Date: 11/8/2006 1:39 AM Calculation Date: 11/8/2006 2:00 AM

Operator : Chen

Workstation:

Instrument : Varian Star \#1

Detector Type: 335

Channel : $1=254 \mathrm{~nm}$

Bus Address : 44

Sample Rate : $10.00 \mathrm{~Hz}$

Run Time : $21.000 \mathrm{~min}$

** LC Workstation Version 6.30 ** 02970-73d0-ae7-0230 **

Chart Speed $=0.93 \mathrm{~cm} / \mathrm{min} \quad$ Attenuation $=37 \quad$ Zero Offset $=3 \%$

Start Time $=0.000 \mathrm{~min}$ End Time $=21.000 \mathrm{~min}$ Min $/$ Tick $=1.00$

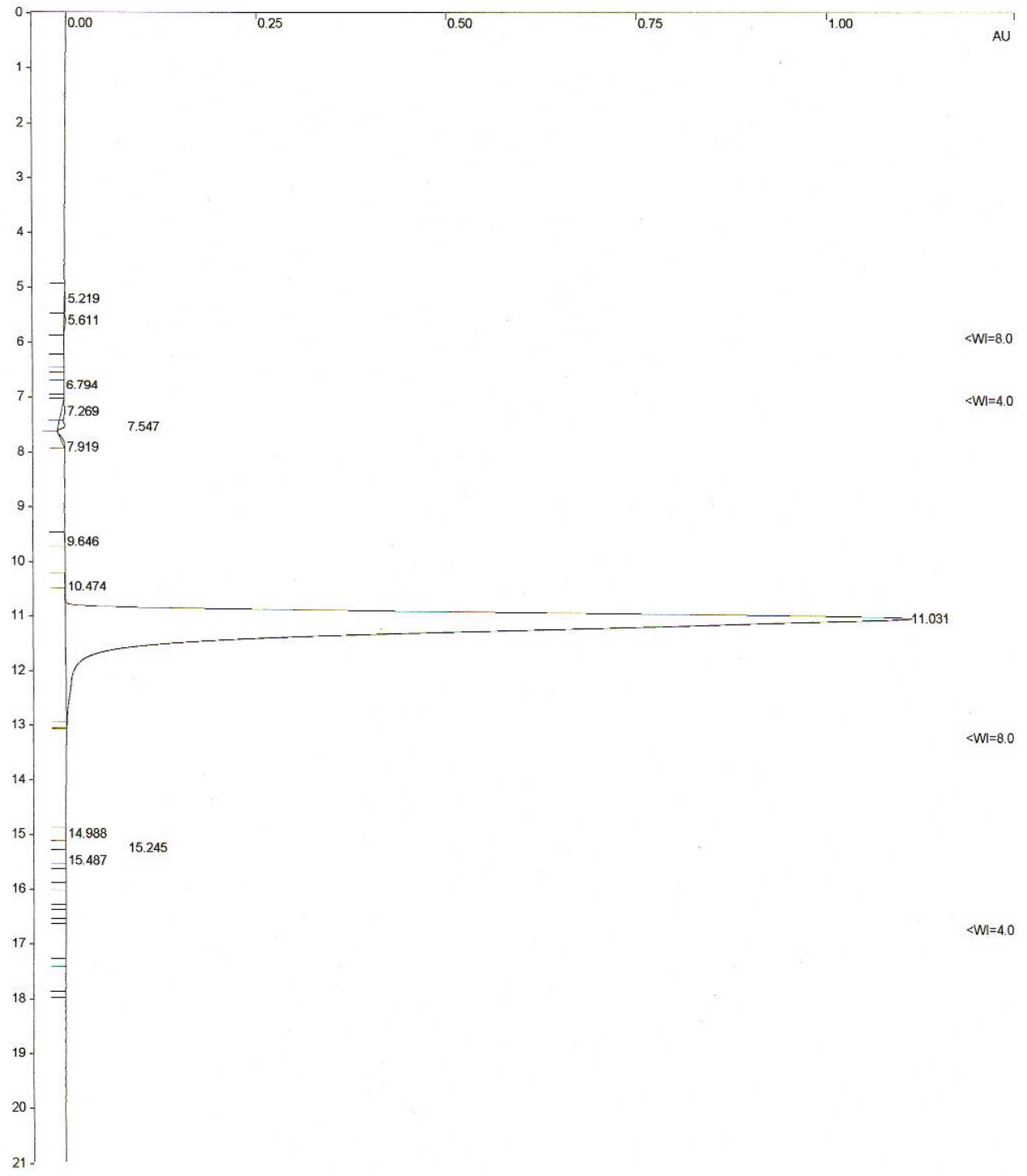




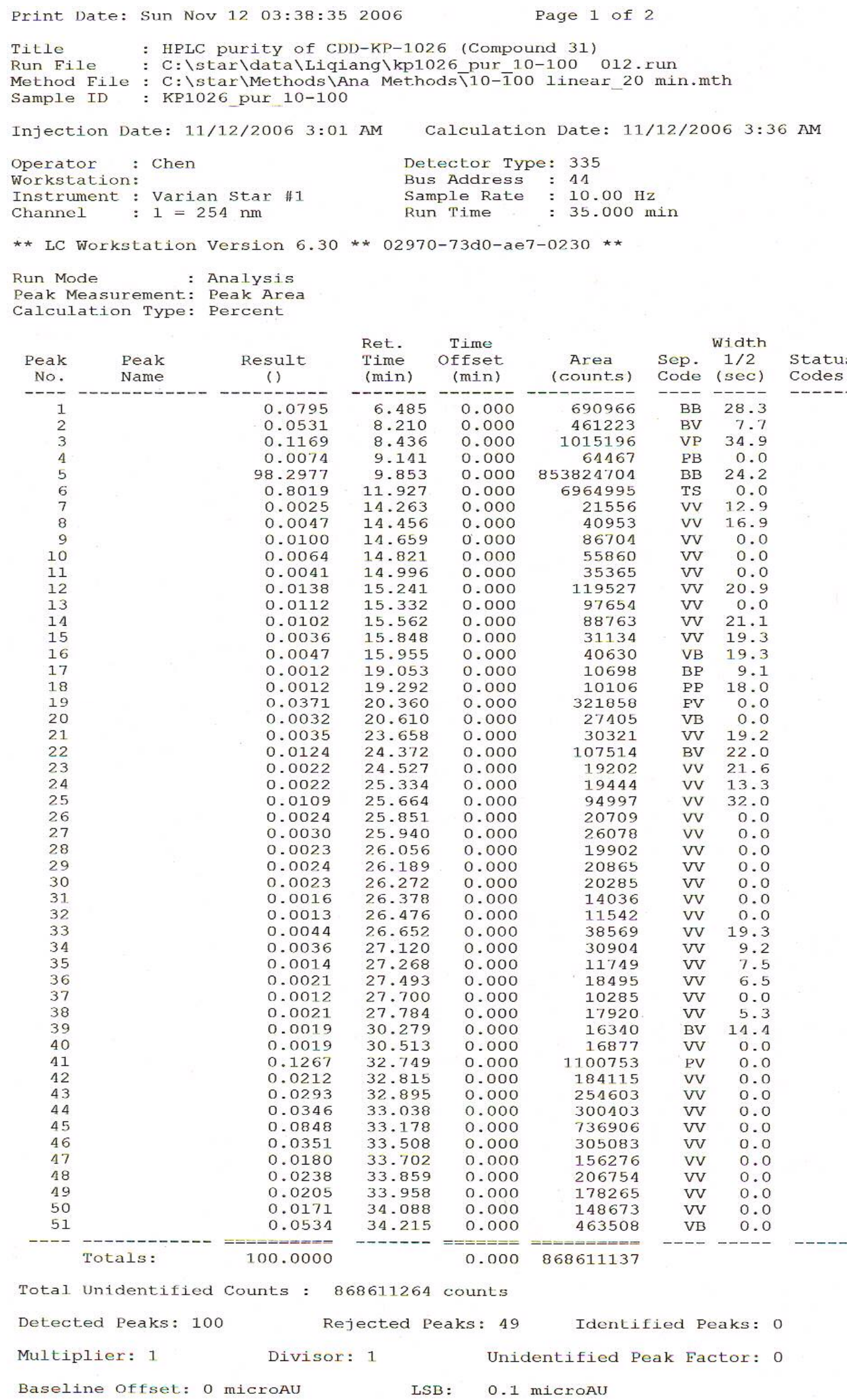


Print Date: Sun Nov 12 03:38:35 2006

Pace 2 of 2

Noise (used): 10 microAU - monitored before this run

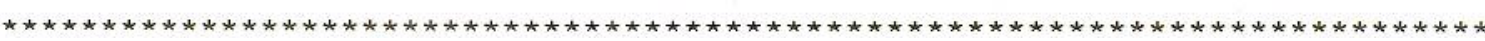


'I'ItLe : HPLC purlty of CDD-KP-1026 (Compound 31)

Run File : C: \star \data \Liqiang $\backslash k p 1026$ pur 10-100 012.run

Method File : C: $\backslash$ star $\backslash$ Methods $\backslash$ Ana Methods $\backslash 10-\overline{1} 00$ linear 20 min.mth

Sample ID : KP1026_pur_10-100

Injection Date: 11/12/2006 3:01 AM Calculation Date: 11/12/2006 3:36 AM

Operator : Chen

Workstation:

Instrument : Varian Star \#1

Detector Type: 335

Channel : $1=254 \mathrm{~nm}$

Bus Address : 44

Sample Rate : $10.00 \mathrm{~Hz}$

Run Time : $35.000 \mathrm{~min}$

** LC Workstation Version 6.30 ** 02970-73d0-ae7-0230 **

Chart Speed $=0.56 \mathrm{~cm} / \mathrm{min} \quad$ Attenuation $=106 \quad$ Zero Offset $=2 \%$

Start Time $=0.000$ min End Time $=35.000$ min Min $/ \mathrm{Tick}=1.00$

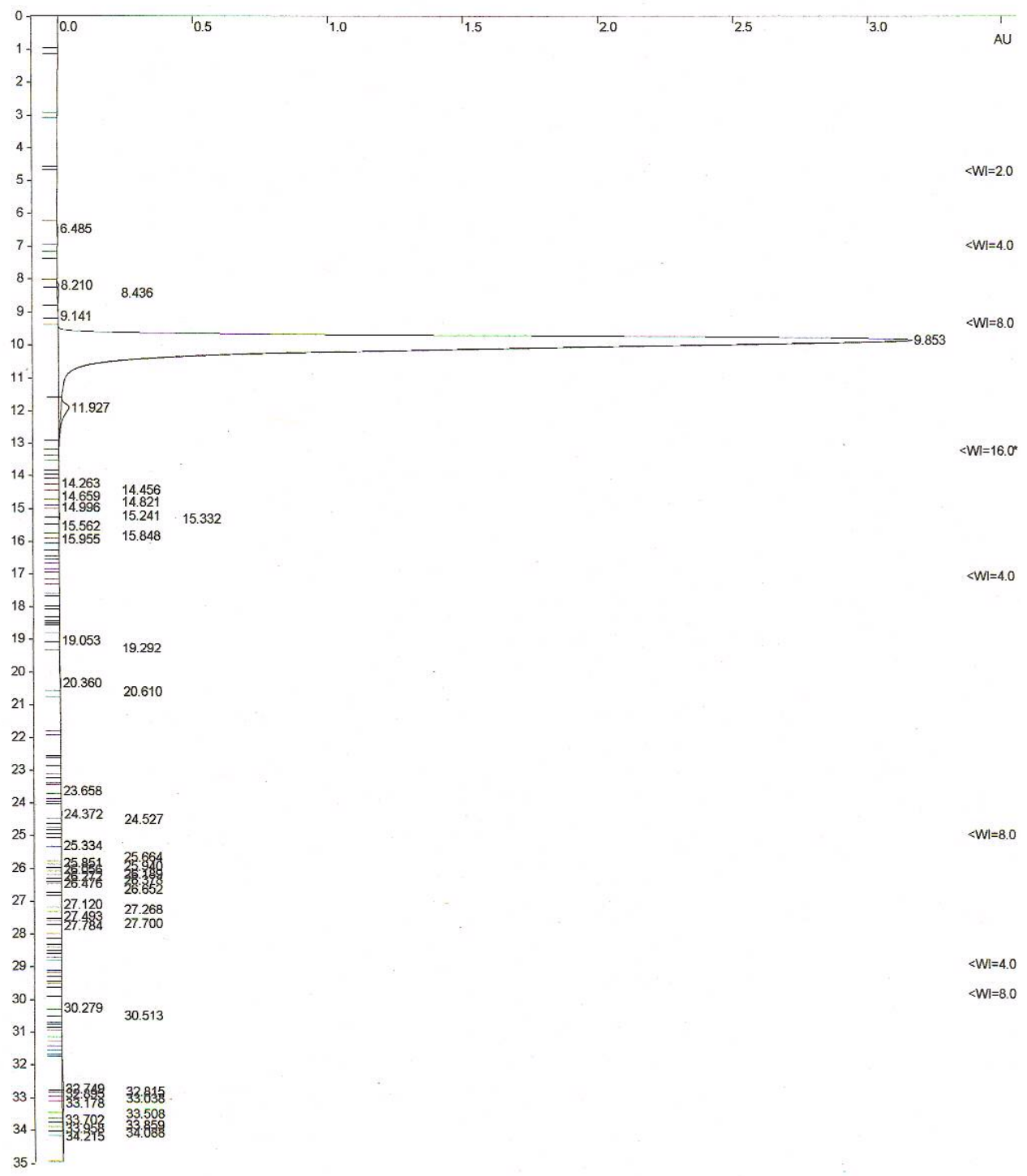




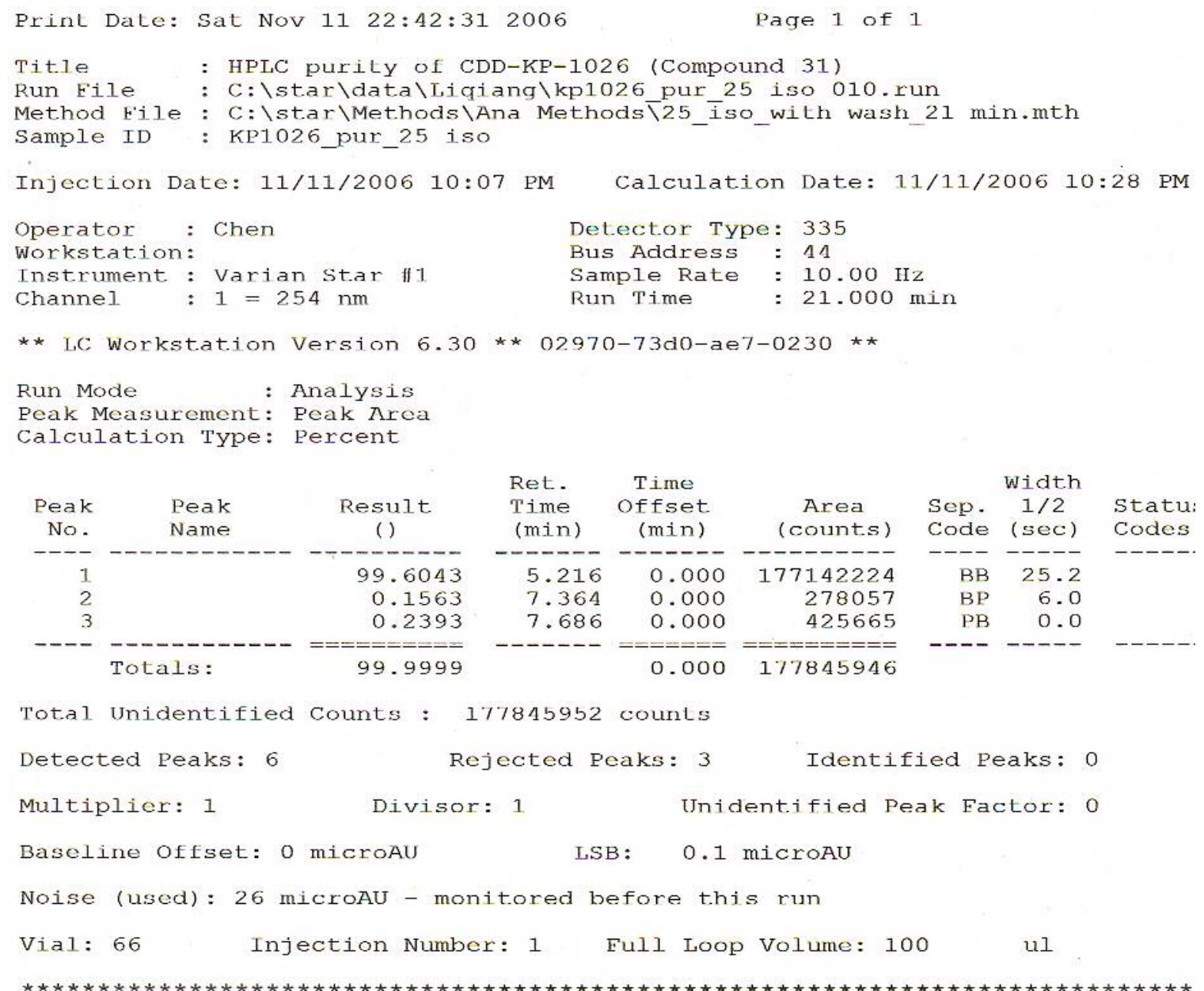


Title

: HPLC purıty of CDD-KP-1026 (Compound 31)

Run Fil

: C: \star \data \Liqiang \kp1026 pur 25 iso 010.run

Method File : C: \star\Methods \Ana Methods $\backslash 25$ iso with wash 21 min.mth

Sample ID : KP1026_pur_25 iso

Injection Date: 11/11/2006 10:07 PM Calculation Date: 11/11/2006 10:28 PM

Operator : Chen

Workstation:

Instrument : Varian star \#1

Channel : $1=254 \mathrm{~nm}$

** LC Workstation Version 6.30 ** 02970-73d0-ae7-0230 **

$\begin{array}{llll}\text { Chart Speed }= & 0.93 \mathrm{~cm} / \mathrm{min} & \text { Attenuation }=21 & \text { Zero Offset }=3 \% \\ \text { Start Time }= & 0.000 \mathrm{~min} & \text { End Time }=21.000 \mathrm{~min} & \text { Min } / \mathrm{Tick}=1.00\end{array}$

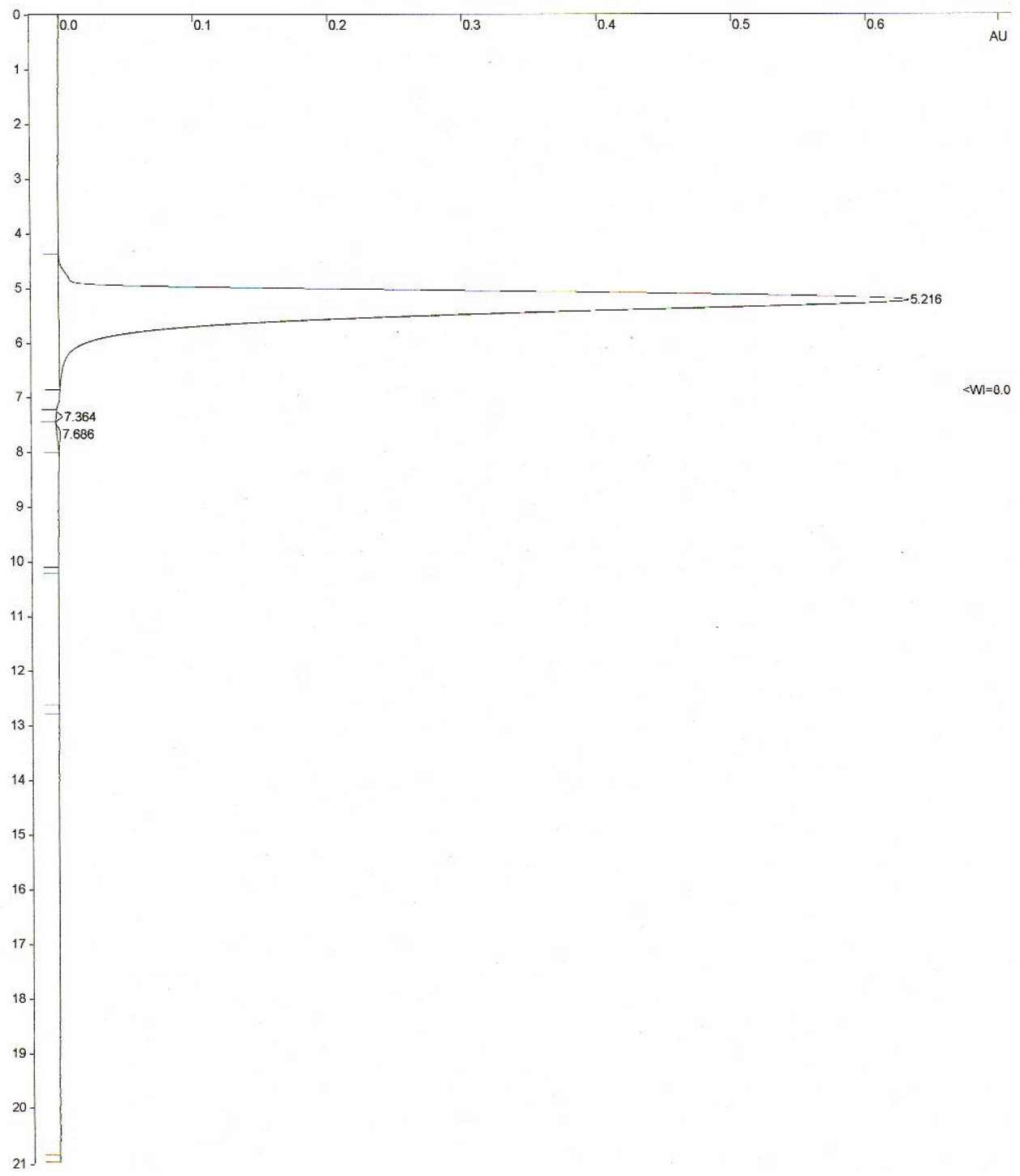




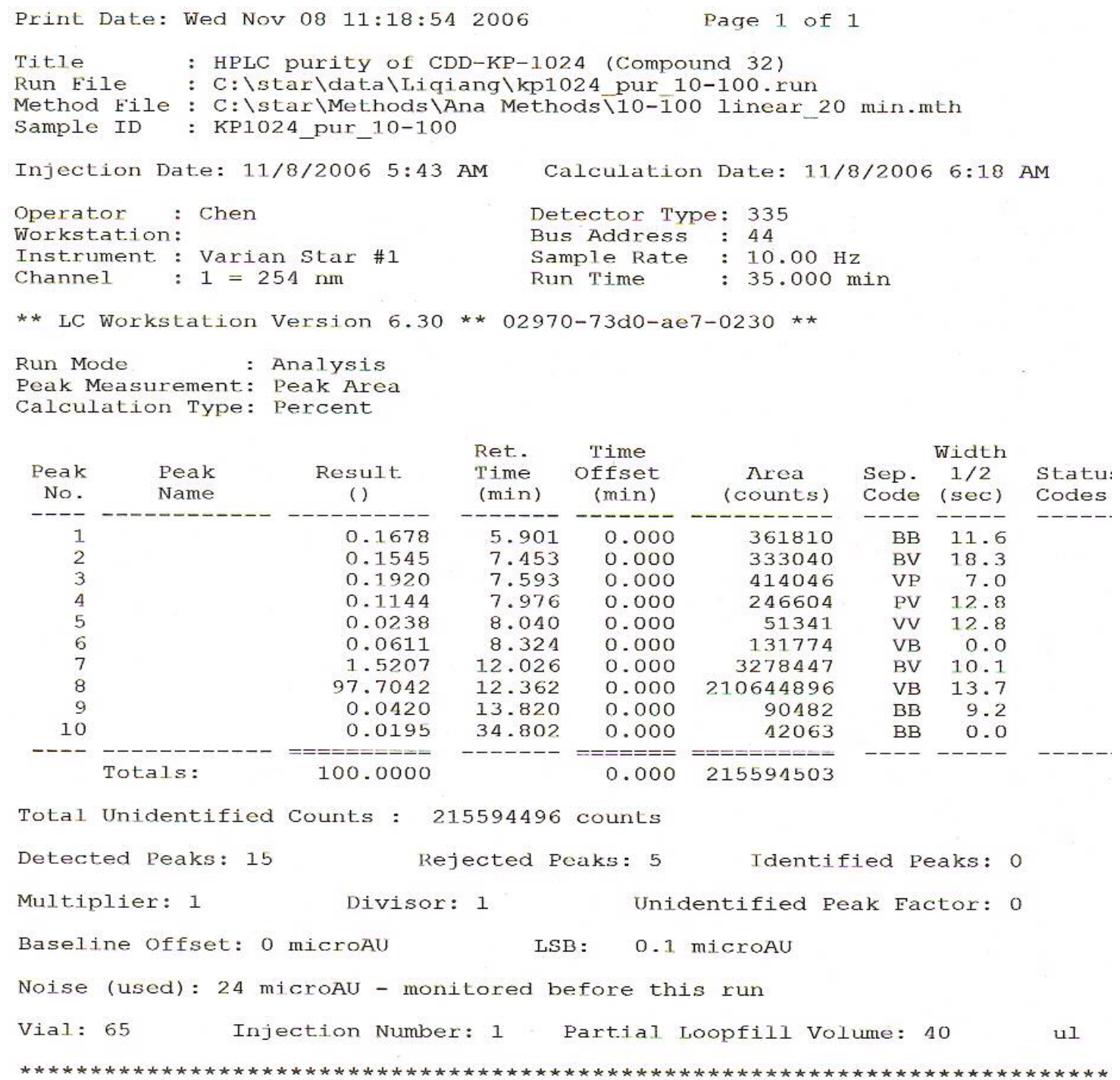


'I'Ite : HPLC purity of CDD-KP-IU24 (Compound 32)

Run File : C: \star \data \Liqiang $\backslash k p 1024$ pur 10-100.run

Method File : C: \star \Methods $\backslash$ Ana Methods $\backslash 10-\overline{1} 00$ linear 20 min.mth

Sample ID : KP1024_pur_10-100

Injection Date: 11/8/2006 5:43 AM Calculation Date: 11/8/2006 6:18 AM

Operator : Chen

Workstation:

Instrument : Varian star \#1

Detector Type: 335

Channel : $1=254 \mathrm{~nm}$

Bus Address : 44

Sample Rate : $10.00 \mathrm{~Hz}$

Run Time : $35.000 \mathrm{~min}$

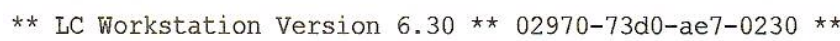

Chart Speed $=0.56 \mathrm{~cm} / \mathrm{min} \quad$ Attenuation $=44 \quad$ Zero offset $=3 \%$

Start Time $=0.000 \mathrm{~min}$ End Time $=35.000 \mathrm{~min} \mathrm{Min} / \mathrm{Tick}=1.00$

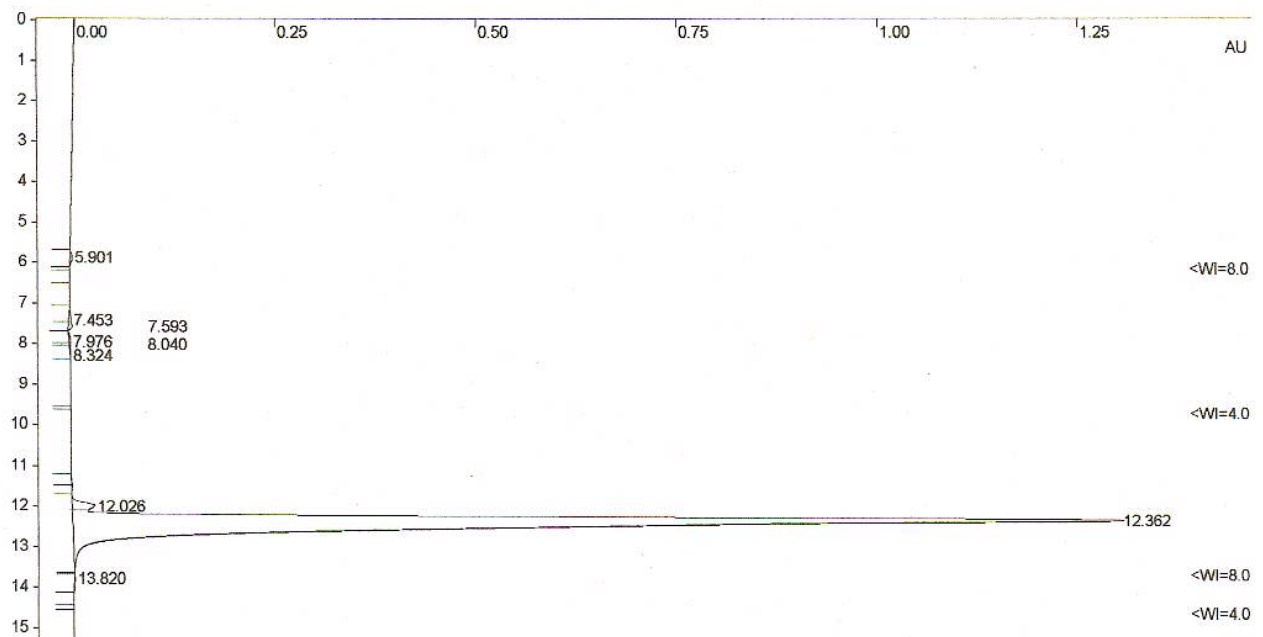




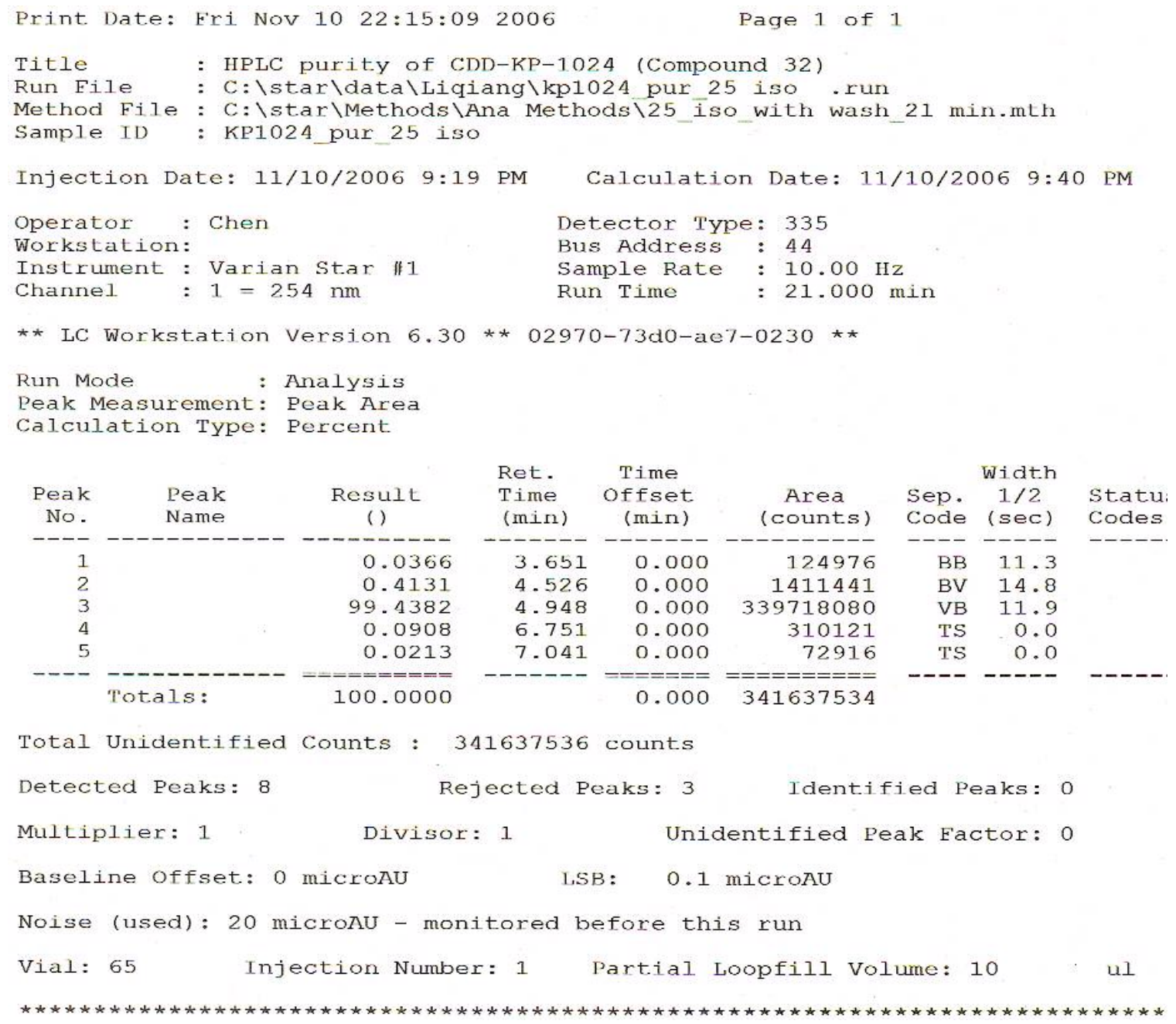


'I'Itle : HPLC purlty of CDD-KP-1024 (Compound 32)

Run File : C: \star\data\Liqiang $\backslash k p 1024$ pur 25 iso .run

Method File : C: \star\Methods \Ana Methods $\backslash 25$ iso with wash 21 min.mth

Sample ID : KP1024_pur_25 iso

Injection Date: 11/10/2006 9:19 PM Calculation Date: 11/10/2006 9:40 PM

Operator : Chen

Workstation:

Instrument : Varian star \#1

Detector Type: 335

Channel

: $1=254 \mathrm{~nm}$

Bus Address : 44

Sample Rate : $10.00 \mathrm{~Hz}$

Run Time : $21.000 \mathrm{~min}$

** LC Workstation Version 6.30 ** 02970-73d0-ae7-0230 **

Chart Speed $=0.93 \mathrm{~cm} / \mathrm{min} \quad$ Attenuation $=72 \quad$ Zero offset $=2 \%$

Start Time $=0.000 \mathrm{~min}$ End Time $=21.000 \mathrm{~min} \mathrm{Min} / \mathrm{Tick}=1.00$

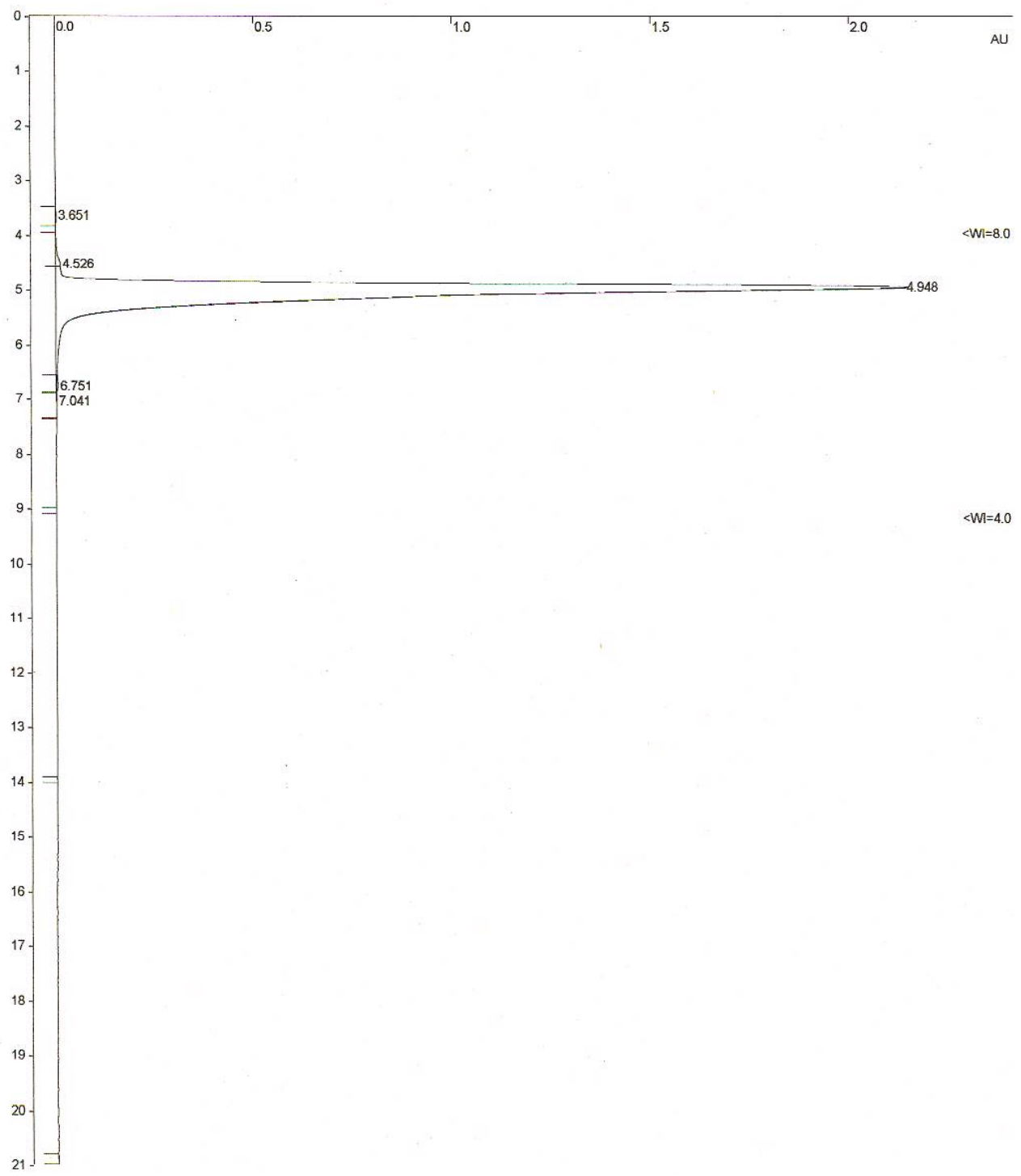




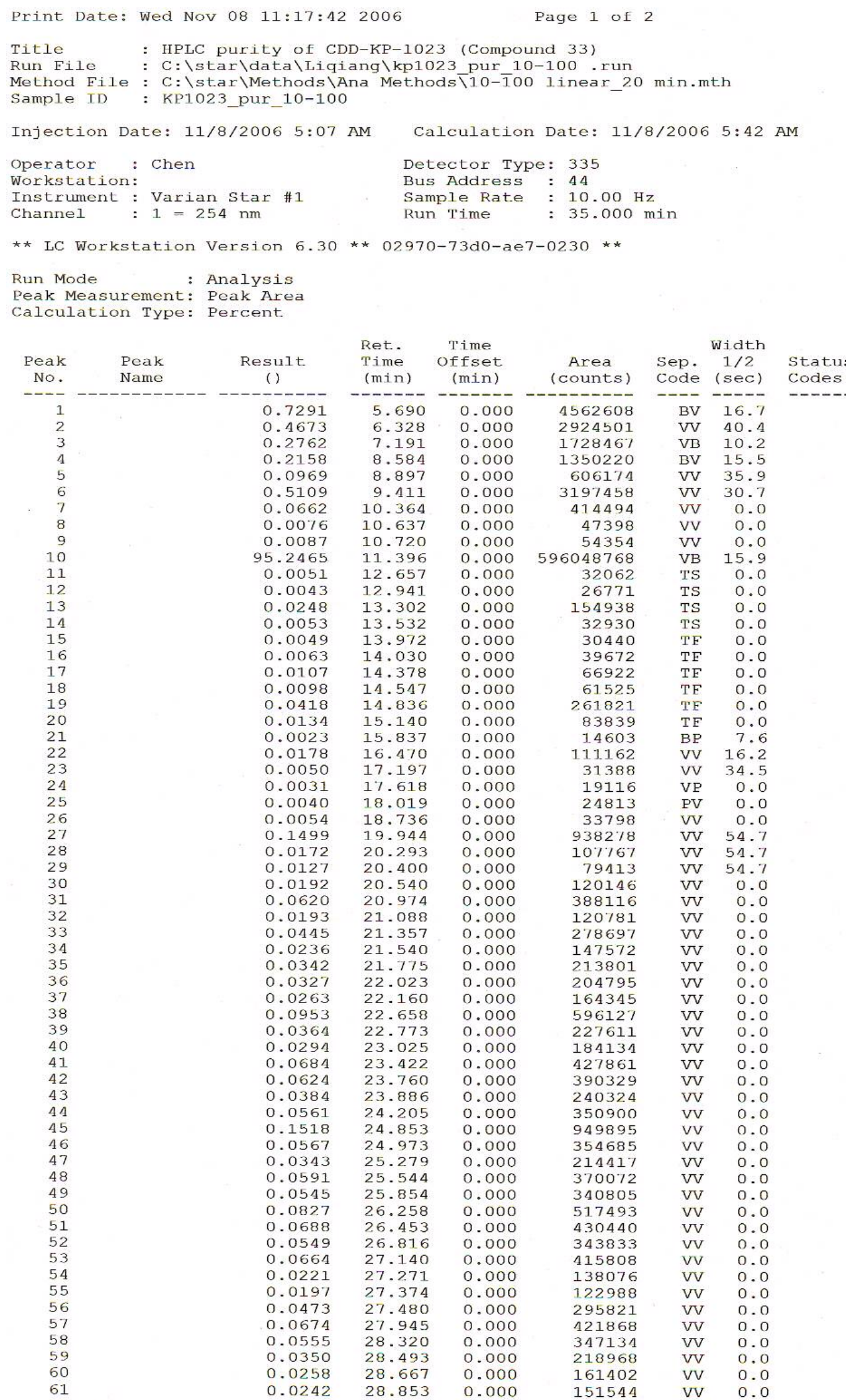


Print Date: Wed Nov o8 11:17:42 2006

\begin{tabular}{rr}
62 & 0.0450 \\
63 & 0.0593 \\
64 & 0.0365 \\
65 & 0.0270 \\
66 & 0.0178 \\
67 & 0.0165 \\
68 & 0.0369 \\
69 & 0.0109 \\
70 & 0.0141 \\
71 & 0.0057 \\
72 & 0.0054 \\
73 & 0.0344 \\
74 & 0.0110 \\
75 & 0.1363 \\
$----0=-10$ \\
\multicolumn{2}{c}{ Totals: }
\end{tabular}

100.0002
Page 2 of 2

$$
\begin{aligned}
& 29.040 \\
& 29.558 \\
& 29.858 \\
& 30.358 \\
& 30.565 \\
& 30.786 \\
& 31.290 \\
& 31.467 \\
& 31.893 \\
& 32.032 \\
& 32.157 \\
& 32.901 \\
& 33.191 \\
& 34.026 \\
& --1 .--
\end{aligned}
$$

$0.000 \quad 281851$

0.000

0.000

0.000

0.000

0.000

0.000

0.000

0.000

0.000

0.000

0.000

0.000

0.000

0.000
371035

228460

169048

111447

103167

231069

68380

88530

35911

33481

215233

68707

853057

625795864

Total Unidentified Counts : 625795776 counts

Detected Peaks: 78

Rejected Peaks: 3

Identified Peaks: 0

Multiplier: 1

Divisor: 1

Unidentified Peak Factor: 0

Baseline offset: 0 microAu

LSB: $\quad 0.1$ microAU

Noise (used) : 17 microAu - monitored before this run

Vial: 64

Injection Number: 1

Partial Loopfill Volume: 10 
TItIe : HPLC purity of CDD-KP-1023 (Compound 33)

Run File : C: \star \data \Liqiang $\backslash k p 1023$ pur 10-100 . run

Method File : C: \star\Methods \Ana Methods $\backslash 10-\overline{1} 00$ linear 20 min.mth

Sample ID : KP1023_pur_10-100

Injection Date: 11/8/2006 5:07 AM Calculation Date: 11/8/2006 5:42 AM

Operator : Chen

Workstation:

Instrument : Varian star \#1

Detector Type: 335

Channel

: $1=254 \mathrm{~nm}$

Bus Address : 44

Sample Rate : $10.00 \mathrm{~Hz}$

Run Time : $35.000 \mathrm{~min}$

** LC Workstation Version $6.30 * \star$ 02970-73d0-ae7-0230 **

Chart Speed $=0.56 \mathrm{~cm} / \mathrm{min} \quad$ Attenuation $=106 \quad$ Zero offset $=2 \%$

Start Time $=0.000 \mathrm{~min}$ End Time $=35.000 \mathrm{~min} \mathrm{Min} / \mathrm{Tick}=1.00$

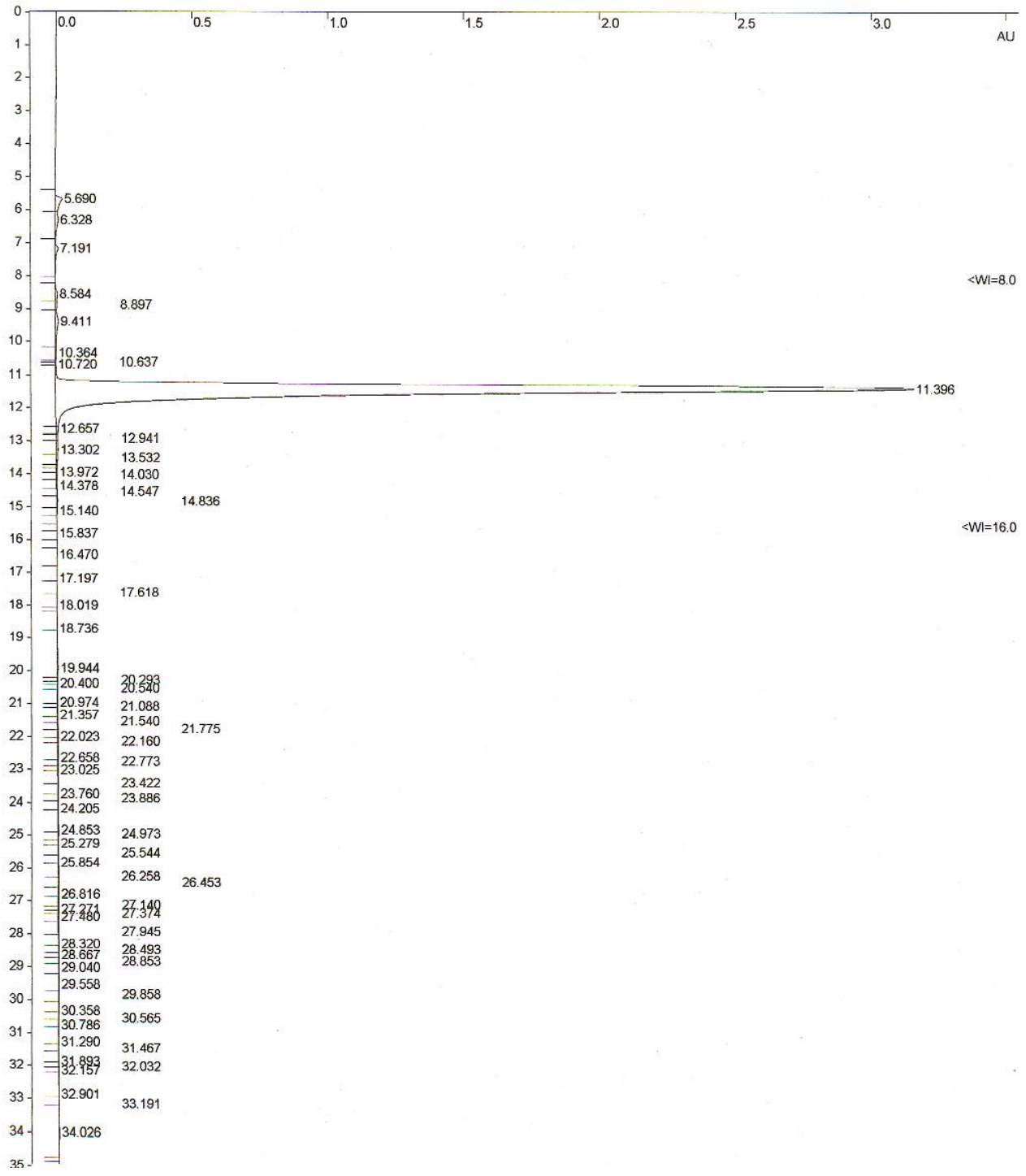




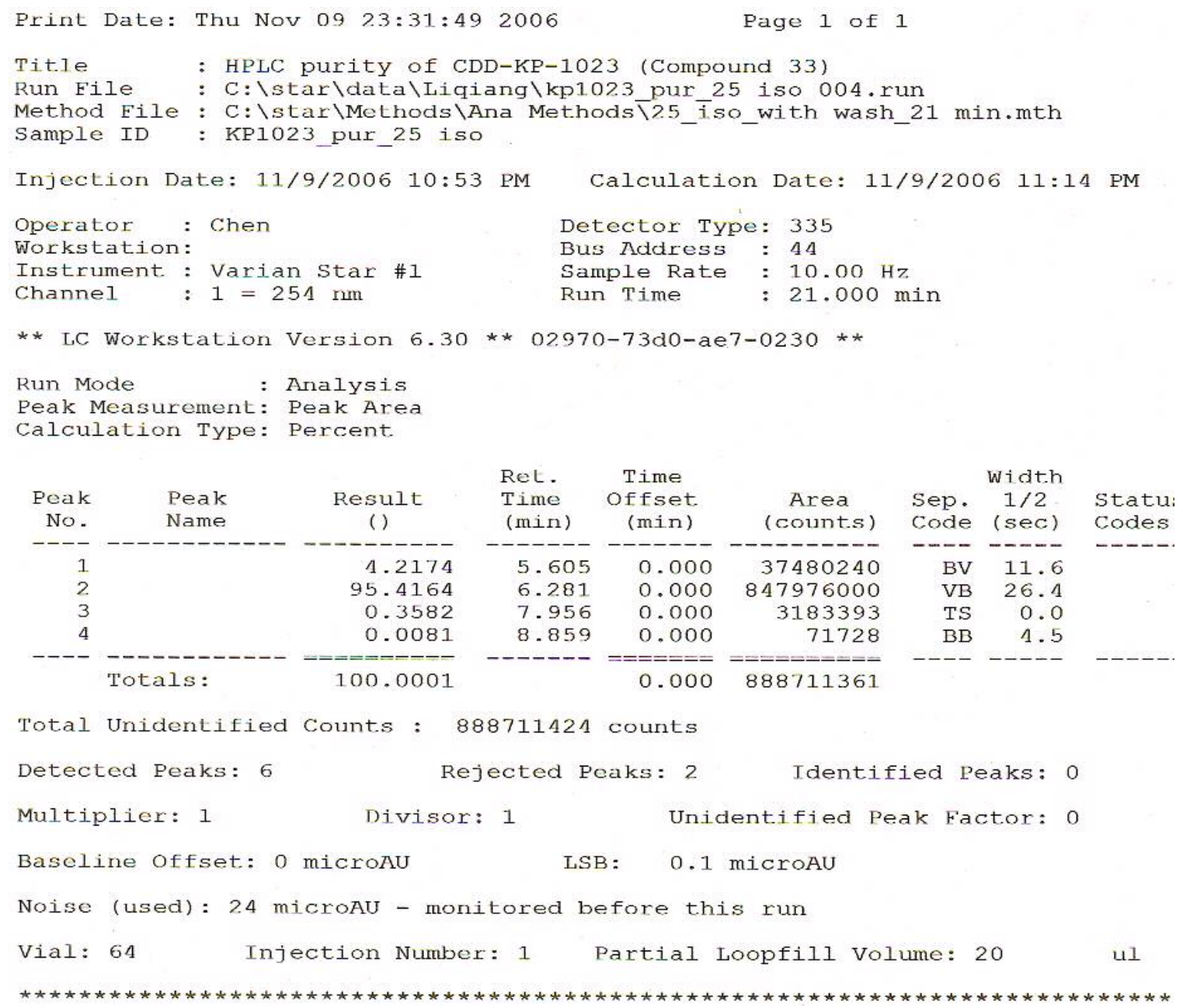


'I'ItLe : HPLC purıty of CDD-KP-1023 (Compound 33)

Run File : C: \star \data \Liqiang $\backslash k p 1023$ pur 25 iso 004.run

Method File : C: \star\Methods \Ana Methods $\backslash 25$ iso with wash 21 min.mth

Sample ID : KP1023_pur_25 iso

Injection Date: 11/9/2006 10:53 PM Calculation Date: 11/9/2006 11:14 PM

Operator : Chen

Workstation:

Instrument : Varian Star \#1

Detector Type: 335

Channel

: $1=254 \mathrm{~nm}$

Bus Address : 44

Sample Rate : $10.00 \mathrm{~Hz}$

** LC Workstation Version 6.30 ** 02970-73d0-ae7-0230 **

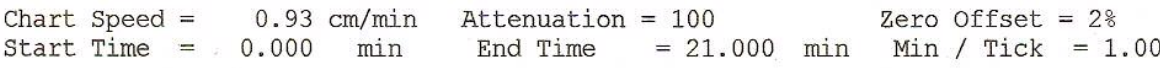

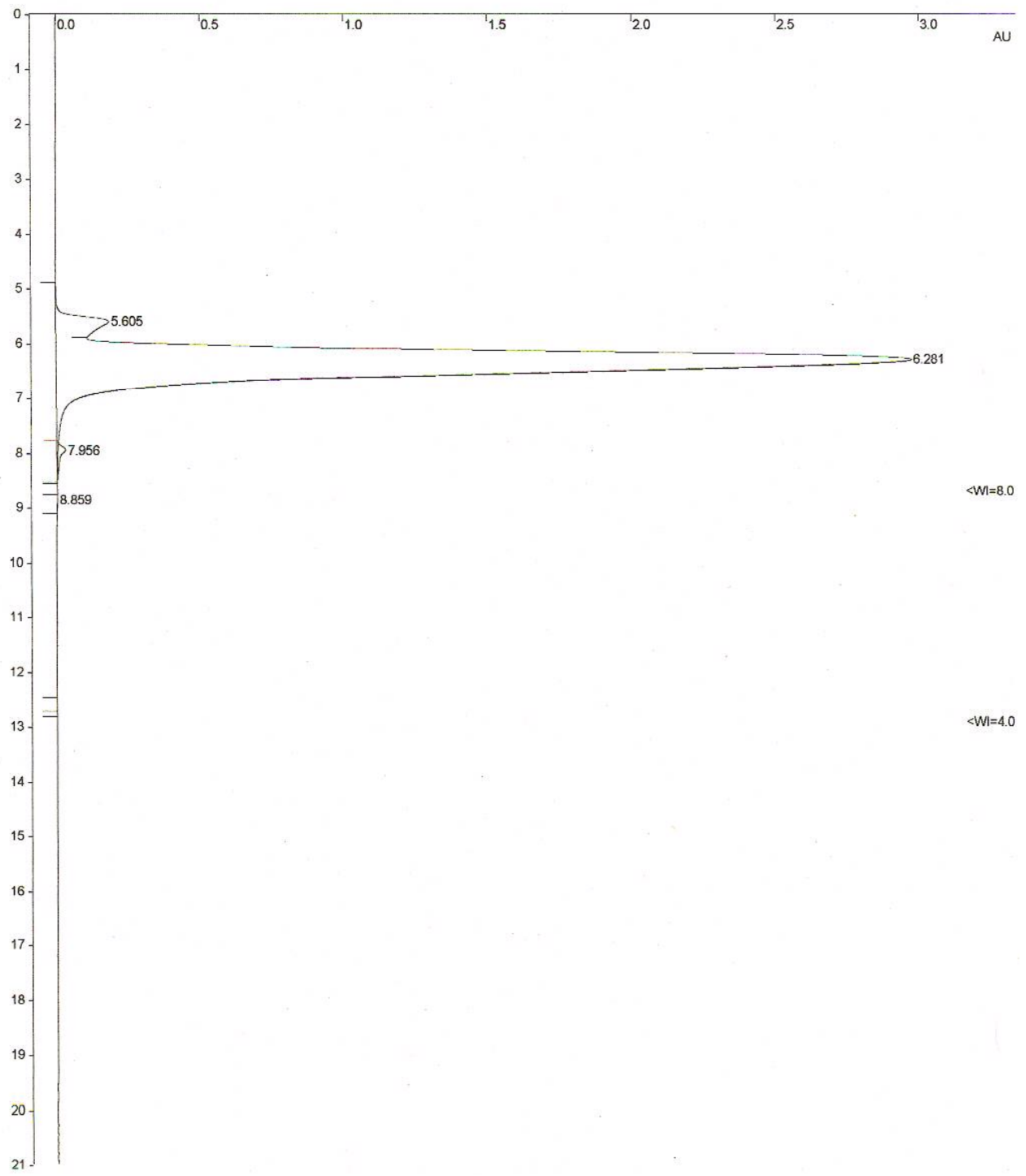


S40

$\begin{array}{llll}\text { Data File: } & \text { c:Istarldatalgaolg55plpuity_45_iso008.ru } & \text { Operator (Calc): } & \text { Chen } \\ \text { Channel: } & \text { 1 }=254 \text { nm RESULTS } & \text { Calc Date: } & \text { 11/30/2006 05:10:59 PM } \\ \text { Sample ID: } & \text { G55plpuity_45_iso } & \text { Times Calculated: } & 2 \\ \text { Operator (Inj): } & \text { Chen } & \text { Calculation Method: } & \text { g55p1puity_45_iso008-1.mth } \\ \text { Injection Date: } & 11 / 30 / 200604: 25: 25 & \text { Instrument (Calc): } & \text { Varian Star \#1 } \\ \text { Injection Method: } & \text { c:lstarlmethodslana methodsl45_iso_with } & \text { Run Mode: } & \text { Analysis } \\ \text { Run Time (min): } & 30.000 & \text { Peak Measurement: } & \text { Peak Area } \\ \text { Workstation: } & & \text { Calculation Type: } & \text { Percent } \\ \text { Instrument (Inj): } & \text { Varian Star \#1 } & \text { Calibration Level: } & \text { N/A } \\ & & \text { Verification Tolerance: N/A }\end{array}$

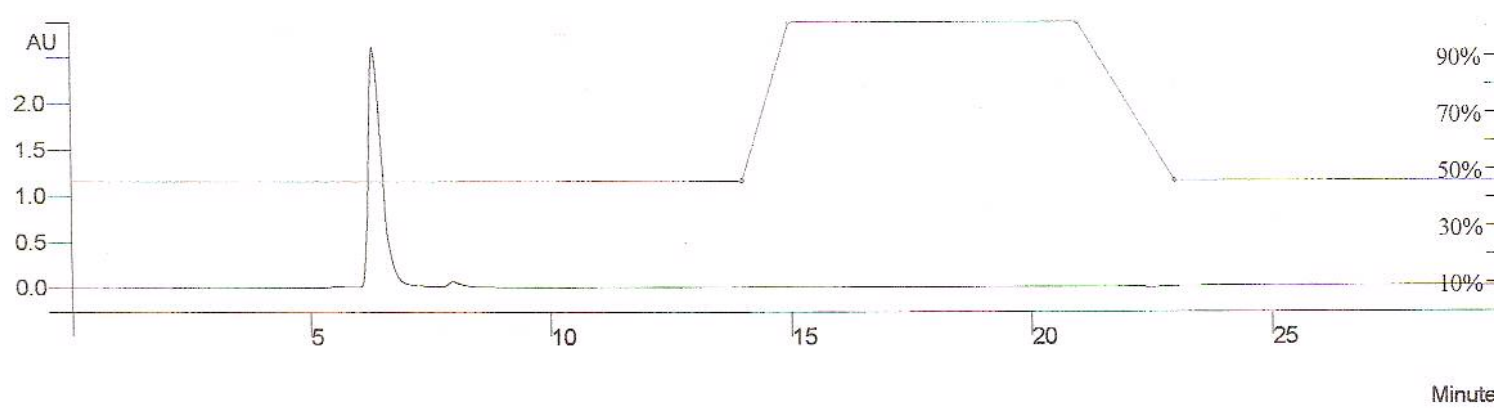

\begin{tabular}{|c|c|c|c|c|c|c|c|c|c|c|}
\hline $\begin{array}{c}\text { Peak } \\
\text { No }\end{array}$ & Peak Name & Result 0 & $\begin{array}{l}\text { Ret. } \\
\text { Time } \\
\text { (min) }\end{array}$ & $\begin{array}{l}\text { Time } \\
\text { Offset } \\
\text { (min) }\end{array}$ & $\begin{array}{c}\text { Area } \\
\text { (counts) }\end{array}$ & $\begin{array}{c}\text { Rel } \\
\text { Ret } \\
\text { Time }\end{array}$ & $\begin{array}{l}\text { Sep. } \\
\text { Code }\end{array}$ & $\begin{array}{c}\text { Width } \\
1 / 2 \\
\text { (sec) }\end{array}$ & $\begin{array}{l}\text { Status } \\
\text { Codes }\end{array}$ & Group \\
\hline 1 & & 0.0466 & 4.970 & 0.000 & 238202 & 0.00 & $\mathrm{BB}$ & 0.0 & & 0 \\
\hline 2 & & 0.2094 & 5.506 & 0.000 & 1069809 & 0.00 & $\mathrm{BB}$ & 0.0 & & 0 \\
\hline 3 & & 97.8275 & 6.300 & 0.000 & 499795168 & 0.00 & BB & 17.5 & & 0 \\
\hline 4 & & 1.9165 & 7.940 & 0.000 & 9791154 & 0.00 & TS & 0.0 & & 0 \\
\hline & & 100.0000 & & 0.000 & 510894336 & & & & & \\
\hline
\end{tabular}

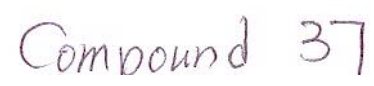


S41

\begin{tabular}{|c|c|c|c|}
\hline Data File: & c:Istarldatalgaolg55p1puity_30_100li.run & Operator (Calc): & Chen \\
\hline Channel: & $1=254 \mathrm{~nm}$ RESULTS & Calc Date: & 11/30/2006 01:07:42 PM \\
\hline Sample ID: & (I55p1puity_30_100li & Times Calculated: & 18 \\
\hline Operator (Inj): & Chen & Calculation Method: & g55plpuity_30_1001i-1.mth \\
\hline Injection Date: & $11 / 30 / 200612: 15: 55$ & Instrument (Calc): & Varian Star \#1 \\
\hline Injection Method: & c: Istarlmethodslana methods $130-100$ & Run Mode: & Analysis \\
\hline Run Time (min): & 30.000 & Peak Measurement: & Peak Area \\
\hline Workstation: & & Calculation Typc: & Pcrcent \\
\hline Instrument (Inj): & Varian Star \#1 & Calibration Level: & $N / A$ \\
\hline & & Verification Tolerance: & N/A \\
\hline
\end{tabular}

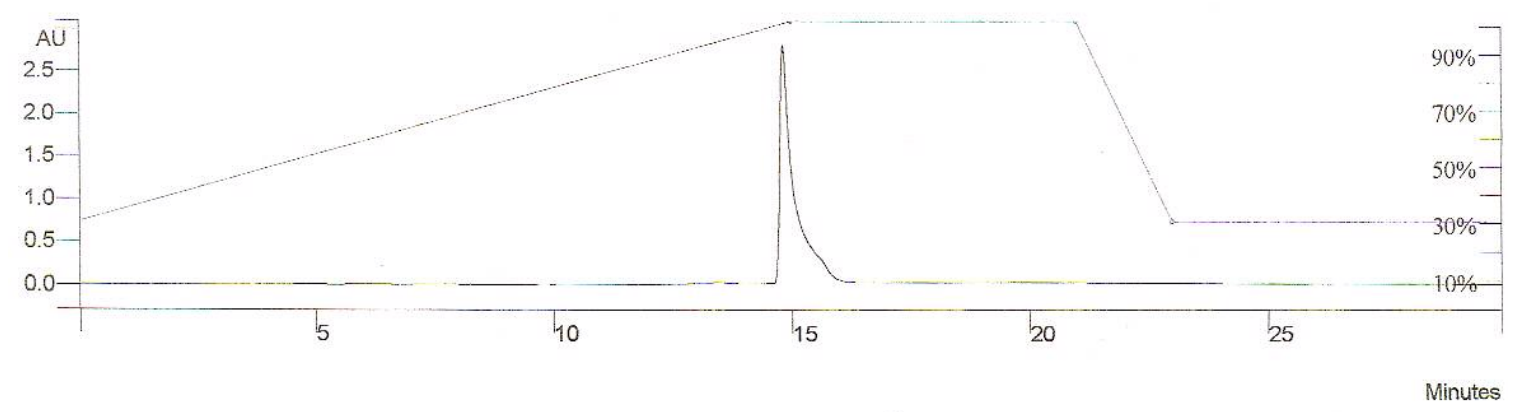

\begin{tabular}{|c|c|c|c|c|c|c|c|c|c|c|}
\hline $\begin{array}{c}\text { Peak } \\
\text { No }\end{array}$ & Peak Name & Result 0 & $\begin{array}{l}\text { Ret. } \\
\text { Time } \\
\text { (min) }\end{array}$ & $\begin{array}{l}\text { Time } \\
\text { Offset } \\
\text { (min) }\end{array}$ & $\begin{array}{c}\text { Area } \\
\text { (counts) }\end{array}$ & $\begin{array}{c}\text { Rel } \\
\text { Ret } \\
\text { Time }\end{array}$ & $\begin{array}{l}\text { Sep. } \\
\text { Code }\end{array}$ & $\begin{array}{l}\text { Width } \\
1 / 2 \\
\text { (sec) }\end{array}$ & $\begin{array}{l}\text { Status } \\
\text { Codes }\end{array}$ & Group \\
\hline 1 & & 0.2773 & 4.802 & 0.000 & 1682407 & 0.00 & VV & 21.9 & & 0 \\
\hline 2 & & 0.1911 & 5.035 & 0.000 & 1159293 & 0.00 & VP & 20.4 & & 0 \\
\hline 3 & & 0.5900 & 5.995 & 0.000 & 3578835 & 0.00 & PV & 0.0 & & 0 \\
\hline 4 & & 0.3010 & 6.387 & 0.000 & 1826096 & 0.00 & VP & 33.4 & & 0 \\
\hline 5 & & 0.1626 & 7.120 & 0.000 & 986568 & 0.00 & PV & 15.0 & & 0 \\
\hline 6 & & 0.6231 & 13.179 & 0.000 & 3780038 & 0.00 & BV & 36.6 & & 0 \\
\hline 7 & & 0.9611 & 13.435 & 0.000 & 5830472 & 0.00 & VV & 44.3 & & 0 \\
\hline 8 & & 96.8077 & 14.825 & 0.000 & 587255232 & 0.00 & $\mathrm{VB}$ & 13.5 & & 0 \\
\hline 9 & & 0.0860 & 17.504 & 0.000 & 521558 & 0.00 & TS & 0.0 & & 0 \\
\hline
\end{tabular}

\section{Compound 37}


S42

$\begin{array}{llll}\text { Data Filc: } & \text { c:Istarldatalgaolg53puity_20_100lin.run } & \text { Operator (Calc): } & \text { Chen } \\ \text { Channel: } & 1=254 \text { nm RESULTS } & \text { Calc Datc: } & 11 / 30 / 200610: 17: 50 \\ \text { Sample II): } & \text { G53puity_20_100lin } & \text { Times Calculated: } & 1 \\ \text { Operator (Inj): } & \text { Chen } & \text { Calculation Method: } & \text { c:Istarlmethodslana methods L20-100 } \\ \text { Injection Date: } & 11 / 30 / 2006 \text { 09:42:47 } & \text { Instrument (Calc): } & \text { Varian Star \#1 } \\ \text { Injection Method: } & \text { c:lstarlmethodslana methods L20-100 } & \text { Run Mode: } & \text { Analysis } \\ \text { Run Time (min): } & 35.000 & \text { Peak Measurement: } & \text { Peak Area } \\ \text { Workstation: } & & \text { Calculation Type: } & \text { Percent } \\ \text { Instrument (Inj): } & \text { Varian Star } \# 1 & \text { Calibration Level: } & \text { N/A } \\ & & \text { Verification Tolerance: N/A }\end{array}$

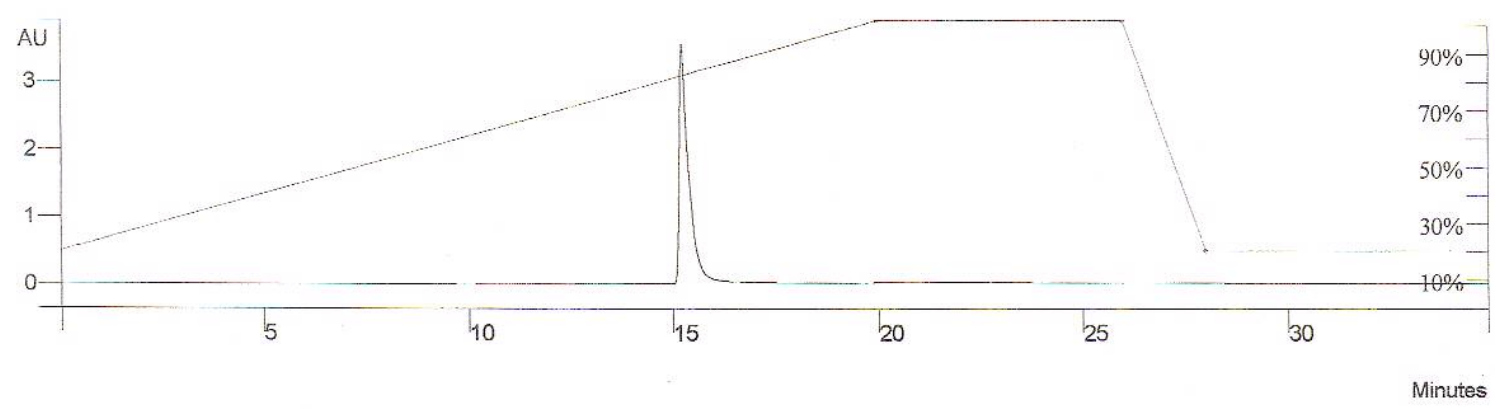

\begin{tabular}{|c|c|c|c|c|c|c|c|c|c|c|}
\hline $\begin{array}{c}\text { Peak } \\
\text { No }\end{array}$ & Peak Name & Result 0 & $\begin{array}{l}\text { Ret. } \\
\text { Time } \\
\text { (min) }\end{array}$ & $\begin{array}{l}\text { Time } \\
\text { Offset } \\
\text { (min) }\end{array}$ & $\begin{array}{c}\text { Area } \\
\text { (counts) }\end{array}$ & $\begin{array}{l}\text { Rel } \\
\text { Ret } \\
\text { Time }\end{array}$ & $\begin{array}{l}\text { Sep. } \\
\text { Code }\end{array}$ & $\begin{array}{c}\text { Width } \\
1 / 2 \\
\text { (sec) }\end{array}$ & $\begin{array}{l}\text { Status } \\
\text { Codes }\end{array}$ & Group \\
\hline 1 & & 0.0301 & 5.506 & 0.000 & 174846 & 0.00 & $\mathrm{BB}$ & 7.5 & & 0 \\
\hline 2 & & 0.0340 & 8.214 & 0.000 & 197891 & 0.00 & $\mathrm{BB}$ & 0.0 & & 0 \\
\hline 3 & & 0.2561 & 9.838 & 0.000 & 1490067 & 0.00 & $\mathrm{BB}$ & 32.6 & & 0 \\
\hline 4 & & 0.1038 & 12.917 & 0.000 & 603773 & 0.00 & BV & 16.6 & & 0 \\
\hline 5 & & 0.1649 & 13.886 & 0.000 & 959138 & 0.00 & VV & 15.9 & & 0 \\
\hline 6 & & 0.0997 & 14.276 & 0.000 & 580156 & 0.00 & VV & 0.0 & & 0 \\
\hline 7 & & 0.0929 & 14.530 & 0.000 & 540595 & 0.00 & VP & 0.0 & & 0 \\
\hline 8 & & 98.6126 & 15.221 & 0.000 & 573719616 & 0.00 & PB & 12.9 & & 0 \\
\hline 9 & & 0.1939 & 21.537 & 0.000 & 1128335 & 0.00 & $\mathrm{BB}$ & 0.0 & & 0 \\
\hline 10 & & 0.1966 & 22.723 & 0.000 & 1143669 & 0.00 & $\mathrm{BB}$ & 13.1 & & 0 \\
\hline 11 & & 0.2154 & 31.691 & 0.000 & 1253102 & 0.00 & $\mathrm{BB}$ & 35.6 & & 0 \\
\hline & & 100.0000 & & 0.000 & 581791232 & & & & & \\
\hline
\end{tabular}

Compound 38 
S43

\begin{tabular}{|c|c|c|c|}
\hline Data File: & c:Istarldatalgaolg53puity_35_iso.run & Operator (Calc): & Chen \\
\hline Channel: & $1=254 \mathrm{~nm}$ RESUL $\mathrm{TS}$ & Calc Date: & $11 / 30 / 200611: 46: 08$ \\
\hline Sample ID: & G53puity_35_iso & Times Calculated: & 2 \\
\hline Operator (Inj): & Chen & Calculation Method: & g53puity_35_iso-1.mth \\
\hline Injection Date: & $11 / 30 / 200611: 10: 18$ & Instrument (Calc): & Varian Star \#1 \\
\hline Injection Method: & c:Istarlmethodslana methodsl35_iso_with & Run Mode: & ヘnalysis \\
\hline Run Time (min): & 30.000 & Peak Measurement: & Peak Area \\
\hline Workstation: & & Calculation Type: & Percent \\
\hline Instrument (Inj): & Varian Star \#1 & Calibration Level: & N/A \\
\hline & & Verification Tolerance: & N/A \\
\hline
\end{tabular}

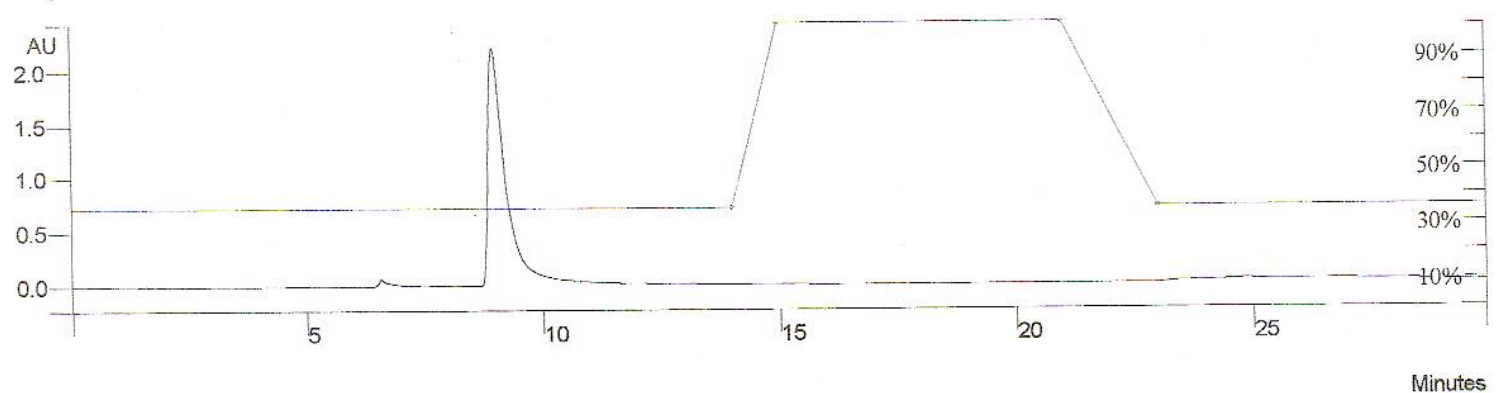

\begin{tabular}{|c|c|c|c|c|c|c|c|c|c|c|}
\hline $\begin{array}{c}\text { Peak } \\
\text { No }\end{array}$ & Peak Name & Result 0 & $\begin{array}{l}\text { Ret. } \\
\text { Time } \\
\text { (min) }\end{array}$ & $\begin{array}{l}\text { Time } \\
\text { Offset } \\
\text { (min) }\end{array}$ & $\begin{array}{c}\text { Arca } \\
\text { (counts) }\end{array}$ & $\begin{array}{c}\text { Rel } \\
\text { Ret } \\
\text { Time }\end{array}$ & $\begin{array}{l}\text { Sep. } \\
\text { Code }\end{array}$ & $\begin{array}{c}\text { Width } \\
1 / 2 \\
\text { (sec) }\end{array}$ & $\begin{array}{l}\text { Status } \\
\text { Codes }\end{array}$ & Group \\
\hline 1 & & 0.0130 & 4.924 & 0.000 & 80295 & 0.00 & $\mathrm{BB}$ & 6.3 & & 0 \\
\hline 2 & & 1.9063 & 6.566 & 0.000 & 11749025 & 0.00 & BB & 11.0 & & 0 \\
\hline 3 & & 98.0807 & 8.947 & 0.000 & 604506688 & 0.00 & $\mathrm{BB}$ & 20.7 & & 0 \\
\hline & & 100.0000 & & 0.000 & 616336000 & & & & & \\
\hline
\end{tabular}

Compound 38 